\title{
Troposphere-Stratosphere Coupling Following Tropospheric Blocking and Extratropical Cyclones
}

\author{
HANNAH E. ATtARD ${ }^{\mathrm{a}}$ AND ANDREA L. LANG \\ Department of Atmospheric and Environmental Sciences, University at Albany, \\ State University of New York, Albany, New York
}

(Manuscript received 18 September 2018, in final form 25 February 2019)

\begin{abstract}
A climatology of the 100 - and $250-\mathrm{hPa} 45^{\circ}-75^{\circ} \mathrm{N}$ zonal-mean meridional eddy heat flux anomaly, hereafter heat flux anomaly, was created to examine its variability following cool-season (i.e., October-April) blocks and extratropical cyclones. The goal is to elucidate the dynamical and environmental differences between synoptic events followed by the most extreme heat flux anomalies. The analysis was conducted with the National Aeronautics and Space Administration's Modern-Era Retrospective Analysis for Research and Applications, version 2 reanalysis. The results show that, on average, European blocks and west Pacific cyclones are followed by positive heat flux anomalies while west Pacific blocks and Atlantic extratropical cyclones are followed by negative heat flux anomalies. However, there was a large range of the 11-day-average heat flux anomaly following the events. Events in each region were further partitioned by their 100-hPa heat flux anomaly for a temporal and spatial analysis of the top and bottom quartile of events. Top-quartile events exhibited a baroclinic wave structure with height from the troposphere through the stratosphere, whereas bottom-quartile events were associated with a barotropic wave structure with height; these structures are significant at the $5 \%$ level. The results suggest that the sign of the heat flux anomaly is not dependent on the location of the synoptic event alone, but that there are common climatological and anomalous wave patterns surrounding the synoptic events that result in positive or negative heat flux anomaly. Regardless of event region, the precursor stratospheric structure is a key indicator in whether an event is followed by positive or negative 100-hPa heat flux anomalies.
\end{abstract}

\section{Introduction}

Persistent tropospheric ridges, or blocks, that precede weak stratospheric regimes are often associated with periods of anomalously positive 100 - or $200-\mathrm{hPa}$ meridional eddy heat flux in the midlatitudes (e.g., Martius et al. 2009; Nishii et al. 2011; Colucci and Kelleher 2015). The positive heat flux suggests the presence of upward wave activity flux (WAF) from the troposphere to the stratosphere, as the zonal-mean meridional eddy heat flux is proportional to the vertical component of the Eliassen-Palm (EP) flux vector (Edmon et al. 1980). Consistent with EP flux theory, the convergence of $\mathrm{WAF}$ in the stratosphere produces an easterly acceleration on the climatological stratospheric westerly winds

\footnotetext{
${ }^{a}$ Current affiliation: National Research Council Postdoctoral Associate/Space Science Division, Naval Research Laboratory, Washington, D.C.
}

Corresponding author: Andrea L. Lang, alang@albany.edu
(Holton 2004) that can lead to a breakdown of the stratospheric polar vortex. When there is sufficient WAF convergence, the $10-\mathrm{hPa} 60^{\circ} \mathrm{N}$ zonal-mean westerly winds can reverse to easterly, resulting in a major sudden stratospheric warming (SSW). The thermal and momentum anomalies associated with SSWs can propagate downward to the troposphere, influencing the sign of the northern annular mode/Arctic Oscillation, storm-track locations, and regional temperature anomalies (Baldwin and Dunkerton 2001). To better understand when an SSW will occur, many studies have investigated the blocking-heat flux-SSW relationship (e.g., Quiroz 1986; Martius et al. 2009; Nishii et al. 2011; Colucci and Kelleher 2015). These studies have suggested that the magnitude and sign of the 100-hPa meridional eddy heat flux anomaly, representative of the anomalous upward WAF, is correlated with the location of tropospheric blocking (e.g., Nishii et al. 2011) and/or whether or not a block precedes an SSW (e.g., Martius et al. 2009; Colucci and Kelleher 2015).

Although previous studies showed a statistical relationship between blocks, heat flux anomalies, and 
SSWs, they have not comprehensively addressed the dynamical difference between blocks that are associated with anomalously positive heat flux and those that are not. There is also a gap in our understanding of the role of extratropical cyclones in inducing upward WAF. While blocks have been the primary focus of climatological studies on the precursors to SSWs, they are not the only extratropical phenomena that have the potential to influence upward WAF. A study of the January 2006 SSW by Coy et al. (2009) emphasized the importance of the synoptic-scale waves (i.e., wavenumbers 4-5) in initiating the troposphere-stratosphere coupling and forcing the SSW. They concluded that synoptic-scale phenomena are important considerations when analyzing troposphere-stratosphere coupling and should not be ignored. In a case study of the January 2013 SSW, Coy and Pawson (2015) further emphasized this fact by showing that an extratropical cyclone in the North Atlantic perturbed the waveguide in such a way to promote a period of upward WAF during the initial period of the SSW.

Though Polvani and Waugh (2004) showed that there is a high correlation between the $100-\mathrm{hPa}$ heat flux anomaly and the strength of the vortex, this correlation is much smaller when considering $300-\mathrm{hPa}$ heat flux. Recent studies have thus suggested that while the anomalous heat flux at $100 \mathrm{hPa}$ originates in the troposphere, its large magnitude is partially the result of the stratosphere (e.g., Birner and Albers 2017; de la Cámara et al. 2017). It is an open question as to when heat flux anomaly that originates in the troposphere actually makes it to, and impacts, the stratospheric circulation.

The main objective of this analysis is to elucidate the dynamical and environmental differences between synoptic events with positive and negative heat flux anomalies, to better understand the subset of synoptic events that can precede SSWs. This objective is motivated by the previous literature that showed that synoptic-scale phenomena are important aspects of tropospherestratosphere coupling (e.g., Coy et al. 2009), but that only a quarter of SSWs are preceded by lower-tropospheric wave disturbances (Birner and Albers 2017). With a focus on the Northern Hemisphere, the goals of the study are as follows:

1) Quantify the distribution of cool-season $100-\mathrm{hPa}$ and 250-hPa heat flux anomalies;

2) Determine the climatological location of blocks and extratropical cyclones that are associated with the largest magnitude of heat flux anomalies (regardless of their association with SSWs); and

3) Compare the synoptic- and planetary-scale structures of the subsets of (i) blocks that occur in similar regions but are followed by opposite signed $100-\mathrm{hPa}$ heat flux anomalies,

(ii) bombs that occur in similar regions but are followed by opposite signed 100-hPa heat flux anomalies, and

(iii) blocks and bombs with the same sign of $100-\mathrm{hPa}$ heat flux anomalies.

The remainder of this paper is as follows: section 2 provides an overview of the dataset used and the methodologies employed in this study. Section 3 explores the variability of cool-season heat flux anomalies. Sections 4 and 5 provide temporal and spatial composite analyses, respectively, of the blocks and extratropical cyclones. Section 6 addresses extratropical cyclones and blocks that occur in sequence, while section 7 concludes with a general summary of the results.

\section{Data and methodology}

\section{a. Data}

This analysis was conducted using the National Aeronautics and Space Administration's (NASA) Modern-Era Retrospective Analysis for Research and Applications, version 2 (MERRA-2; Gelaro et al. 2017). The MERRA-2 dataset is an updated version of the original MERRA dataset, which is a fixed assimilation system that utilizes the GEOS-5 atmospheric data assimilation system (Rienecker et al. 2011). The MERRA-2 dataset uses an upgraded version of the GEOS-5 data assimilation system to incorporate modern hyperspectral radiance, microwave observations, and NASA's ozone observations after 2005. The MERRA-2 dataset has a horizontal resolution of $0.625^{\circ} \times 0.5^{\circ}$ and vertical resolution of 72 layers up to $0.01 \mathrm{hPa}$, which is appropriate for analyzing both synoptic events and stratospheric variability.

The study utilizes the MERRA-2 dataset from 19802015 for the cool season, defined as October to April, analyzing 6-hourly output interpolated to pressure surfaces from 1000 to $1 \mathrm{hPa}$ (Global Modeling and Assimilation Office 2015). Unless otherwise indicated, anomalies are taken with respect to a 35-yr (19802015) climatological mean with a 21-day running mean applied to remove the seasonal cycle. Standardized anomalies are calculated based on this same period utilizing the 35-yr standard deviation.

\section{b. Identification of blocks and extratropical cyclones}

Blocks were identified with the Tibaldi and Molteni (1990) blocking definition which utilizes the geopotential height gradient at $500 \mathrm{hPa}$ to identify blocked longitudes. 
TABLE 1. All blocks and bombs partitioned by location.

\begin{tabular}{|c|c|c|c|c|c|c|c|}
\hline & $\begin{array}{l}\text { Northern } \\
\text { Hemisphere }\end{array}$ & $\begin{array}{l}\text { Western Pacific } \\
\left(120^{\circ}-179^{\circ} \mathrm{E}\right)\end{array}$ & $\begin{array}{c}\text { Eastern Pacific } \\
\left(180^{\circ}-121^{\circ} \mathrm{W}\right)\end{array}$ & $\begin{array}{l}\text { North America } \\
\left(120^{\circ}-59^{\circ} \mathrm{W}\right)\end{array}$ & $\begin{array}{l}\text { Atlantic } \\
\left(60^{\circ}-1^{\circ} \mathrm{W}\right)\end{array}$ & $\begin{array}{c}\text { Europe } \\
\left(0^{\circ}-59^{\circ} \mathrm{E}\right)\end{array}$ & $\begin{array}{c}\text { Asia } \\
\left(60^{\circ}-119^{\circ} \mathrm{E}\right)\end{array}$ \\
\hline Blocks & 288 & 54 & 50 & 22 & 63 & 77 & 22 \\
\hline Bombs & 1707 & 676 & 337 & 310 & 328 & 24 & 32 \\
\hline $\begin{array}{l}\text { No. of blocks following } \\
\text { a bomb (\% of all blocks } \\
\text { identified in that region) }\end{array}$ & $183(63.5 \%)$ & $27(50.0 \%)$ & $39(78.0 \%)$ & $15(68.18 \%)$ & $45(71.43 \%)$ & $55(71.43 \%)$ & $2(9.1 \%)$ \\
\hline $\begin{array}{l}\text { No. of bombs preceding } \\
\text { a block ( } \% \text { of all bombs } \\
\text { identified in that region) }\end{array}$ & $329(19.3 \%)$ & $125(18.5 \%)$ & $21(6.2 \%)$ & $74(23.9 \%)$ & 98 (29.9\%) & $7(29.2 \%)$ & $4(12.5 \%)$ \\
\hline
\end{tabular}

Additional constraints were included to combine instantaneous blocked longitudes into coherent blocking events, as outlined in Attard and Lang (2019). These constraints include that blocks must span $\geq 20^{\circ}$ longitude, exist for $\geq 4$ days, and overlap $\geq 10^{\circ}$ at each time step. Cases were then listed in order of strength, as quantified by the magnitude of the geopotential height gradient to the south of the block latitude, and cases that occurred within 4 days and $60^{\circ}$ longitude of a stronger case were removed to prevent the double counting of cases in the temporal analysis. The final case-list has 288 blocks, the locations of which are outlined in Table 1.

Extratropical cyclones were selected by first identifying all extratropical cyclone tracks that formed poleward of $30^{\circ} \mathrm{N}$, lasted $\geq 2$ days, and traversed $\geq 1000 \mathrm{~km}$ using the Hodges $(1994,1995)$ cyclone tracking algorithm. The extratropical cyclones that rapidly intensified and reached the bomb threshold of having a sea level pressure (SLP) decrease of at least $24 \mathrm{hPa}$ in $24 \mathrm{~h}$ with respect to $60^{\circ} \mathrm{N}$ (Sanders and Gyakum 1980) were retained for this analysis. More information on the methodology for identifying bombs can be found in Attard and Lang (2019). The 2852 identified bombs were then listed in order of strength (i.e., the maximum 24-h SLP change) and any bomb that occurred within 4 days and $60^{\circ}$ longitude of a stronger event was not considered in this analysis. The final case list of bombs includes 1707 cases. The locations of the bombs are outlined in Table 1.

\section{c. Quantifying troposphere-stratosphere interaction}

Typically $100 \mathrm{hPa}$ and the latitude range of $45^{\circ}-75^{\circ} \mathrm{N}$ have been used in studies to indicate periods of active upward wave coupling between the troposphere and the stratosphere (e.g., Polvani and Waugh 2004). For this analysis, the troposphere-stratosphere interaction is quantified by the 250 and $100 \mathrm{hPa} 45^{\circ}-75^{\circ} \mathrm{N}$ zonal-mean meridional eddy heat flux, $\left[v^{*} T^{*}\right]$, hereafter heat flux, where the brackets and asterisks indicate the zonal mean and departures from the zonal mean, respectively. The anomalous component of the heat flux calculated as a departure from the climatological mean, $\left[v^{*} T^{*}\right]_{a}$, hereafter heat flux anomaly, is used to diagnose the anomalous wave activity entering the stratosphere. The standardized anomaly, $\left[v^{*} T^{*}\right]_{\sigma}$, is also utilized to make a direct comparison of the heat flux at different levels, as the range of heat flux anomaly changes with pressure level. Specifically, the $250-\mathrm{hPa}$ heat flux anomaly and the $250-\mathrm{hPa}$ standardized heat flux anomaly are calculated to analyze a level close to the tropopause, which provides information to assess whether the heat flux anomaly at $100 \mathrm{hPa}$ originated in the troposphere.

To understand the interaction between climatological and anomalous waves in producing heat flux, the heat flux anomaly can be decomposed following Nishii et al. (2009):

$$
\left[v^{*} T^{*}\right]_{a}=\underbrace{\left[v_{c}^{*} T_{a}^{*}\right]}_{(\mathrm{i})}+\underbrace{\left[v_{a}^{*} T_{c}^{*}\right]}_{(\mathrm{ii})}+\underbrace{\left[v_{a}^{*} T_{a}^{*}\right]_{a}}_{(\text {iii) }} .
$$

In Eq. (1) the brackets and stars are as previously noted and the subscripts $c$ and $a$ refer to the climatological mean and anomaly, respectively. Terms (i) and (ii) in Eq. (1) represent contributions to the heat flux anomaly associated with the interaction between climatological waves and the anomalous waves while term (iii) represents contributions to the heat flux anomaly due solely to anomalies associated with the momentum and thermal fields within the Rossby waves. When calculating the terms of Eq. (1), the raw climatological mean is used (i.e., without the 21-day running mean applied).

The methodology of identifying blocks and analyzing their associated tropopause heat flux anomaly is derived from Nishii et al. (2011). Nishii et al. (2011) calculated the heat flux anomaly following the 30 strongest blocking high events in Europe and the west Pacific from $1979 / 80$ to $2007 / 08$. The blocking strength threshold included in this study is not as stringent as the strength threshold in Nishii et al. (2011) in order to ensure a 
case-list that captures the variety of strong blocks that occur in the Northern Hemisphere. This analysis also does not impose a criterion to consider the same number of cases per region in order to capture the natural preference for high-latitude blocks to develop in the European region.

To explore the horizontal and vertical wave structure of the flow associated with blocks and bombs with the most extreme heat flux anomalies, event-centered composites were calculated by shifting the gridded data for events that occurred in the same region to the mean location of events in that region. To account for differences in longitude spacing with latitude, prior to compositing, the latitude was scaled by the cosine of latitude. Nonevent centered composites are also shown to analyze the zonal asymmetries in the terms on the right-hand side of Eq. (1). The type of composite used (i.e., event centered or not) is noted in the figure captions.

The analysis presented discusses heat flux anomaly and not the full heat flux. Although many events identified in this analysis are followed by short-lived negative heat flux anomaly, the 11-day average of the full heat flux following each event is positive, indicating upward wave activity. A negligible amount of events in this analysis (i.e., $\leq 0.2 \%$ ) had a negative 11 -day-average heat flux at 100 or $250 \mathrm{hPa}$, which would suggest downward wave activity. For blocks, the 11-day average includes and follows the first date of the identified block. For bombs, the 11-day average includes and follows the date of the onset of the maximum 24-h SLP decrease. Shorter averaging periods were calculated with similar conclusions but not included here.

Statistical significance tests were conducted utilizing a bootstrap resampling method repeated 10000 times. First, a sample of cases equal to the number of cases in the group being tested was randomly selected. These 10000 random samples are then utilized to create the expected $95 \%$ confidence interval of the variable. If the mean of the group fell outside of the $95 \%$ confidence interval, it is determined to be statistically significant at the $5 \%$ level. Similar bootstrap resampling methods are employed to test the statistical difference between variables.

\section{Cool-season variability of heat flux anomaly}

\section{a. Intraseasonal variability}

The probability distribution function (PDF), calculated as 25-bin histograms, of the 11-day-average $100-\mathrm{hPa}$ heat flux anomaly following all cool-season days from the 1980/81-2014/15 period has an approximate normal distribution with a small positive skew (Fig. 1a, solid black curve). For the autumn transition season, the November PDF has a standard deviation that is more than double the October PDF (Fig. 1a, solid blue and purple curves, respectively), revealing that November has more variability in heat flux anomaly than October. The PDF of the combined midwinter months of December, January, and February (Fig. 1b, solid yellow curve) has a standard deviation that is $25 \%$ larger than the standard deviation of all cool-season days, with the most extreme heat flux anomalies occurring in January and February (Fig. 1b, solid blue and green curves, respectively). For the spring transition season of March and April, the March PDF is reminiscent of the midwinter PDFs, as it has a similar standard deviation (Fig. 1c, solid purple curve). The intraseasonal variability in $100-\mathrm{hPa}$ heat flux anomalies shown in Fig. 1 are consistent with the analysis of Díaz-Durán et al. (2017), which showed that extreme stratospheric vortex regimes are preceded by the largest $100-\mathrm{hPa}$ heat flux anomalies in January and February and the smallest heat flux anomalies in October, November, and December.

At $250-\mathrm{hPa}$, the 11-day-average heat flux anomaly following all cool-season days and for the combined October and November months are similar to the 100-hPa PDFs, though with a smaller standard deviation (Fig. 1a, dashed curves). Also similar to $100 \mathrm{hPa}$, the most extreme heat flux anomalies at $250 \mathrm{hPa}$ occur in January and February, with the combined midwinter months of December, January, and February having the largest standard deviation of all the periods (Fig. 1b, dashed curves). Interestingly, for the spring transition season, the 250-hPa April standard deviation only decreases by about $30 \%$ from the March value, whereas at $100 \mathrm{hPa}$, the April heat flux anomaly standard deviation is nearly half of the March value (Fig. 1c). The seasonality of the heat flux anomaly at 250 and $100 \mathrm{hPa}$ are similar, suggesting that WAF from the troposphere plays an important role in the seasonality of the lowerstratosphere heat flux anomaly. However, the variability in heat flux anomaly of the lower stratosphere (i.e., $100 \mathrm{hPa}$ ) exceeds corresponding variability near the tropopause (i.e., $250 \mathrm{hPa}$ ), suggesting that stratospheric dynamics may play a role in enhancing the 100-hPa heat flux anomalies (e.g., Birner and Albers 2017; de la Cámara et al. 2017).

The box-and-whisker plots of the 11-day-average 100-hPa heat flux anomaly following blocks and bombs are shown with reference to the \pm 1 standard deviation threshold for each month (Fig. 1a). The interquartile range of the heat flux anomalies following blocks and bombs that occur in October, December, January, March, and April all fall within \pm 1 standard deviation of their respective month's mean. Blocks that occurred in 
(a)

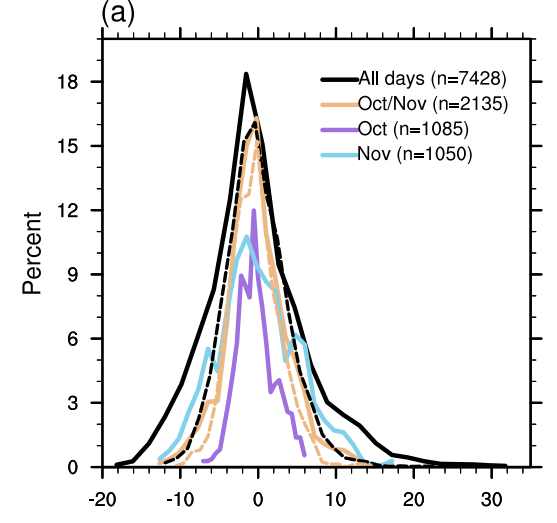

(b)

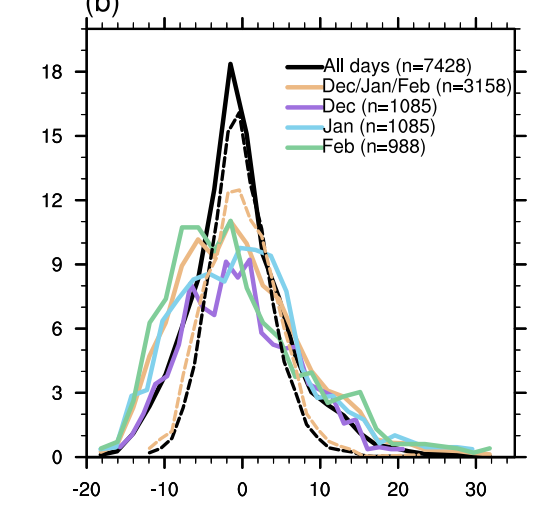

(c)

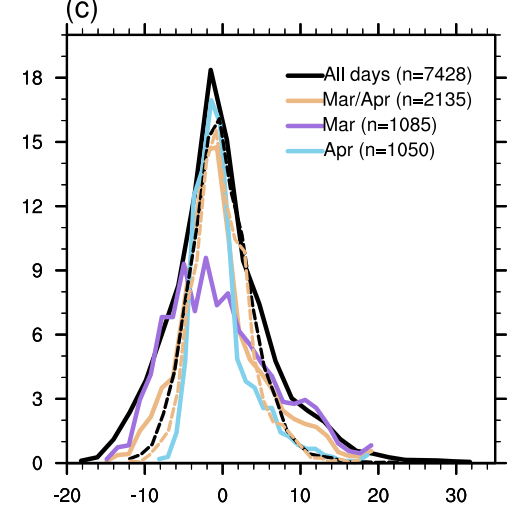

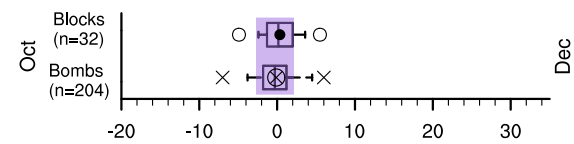
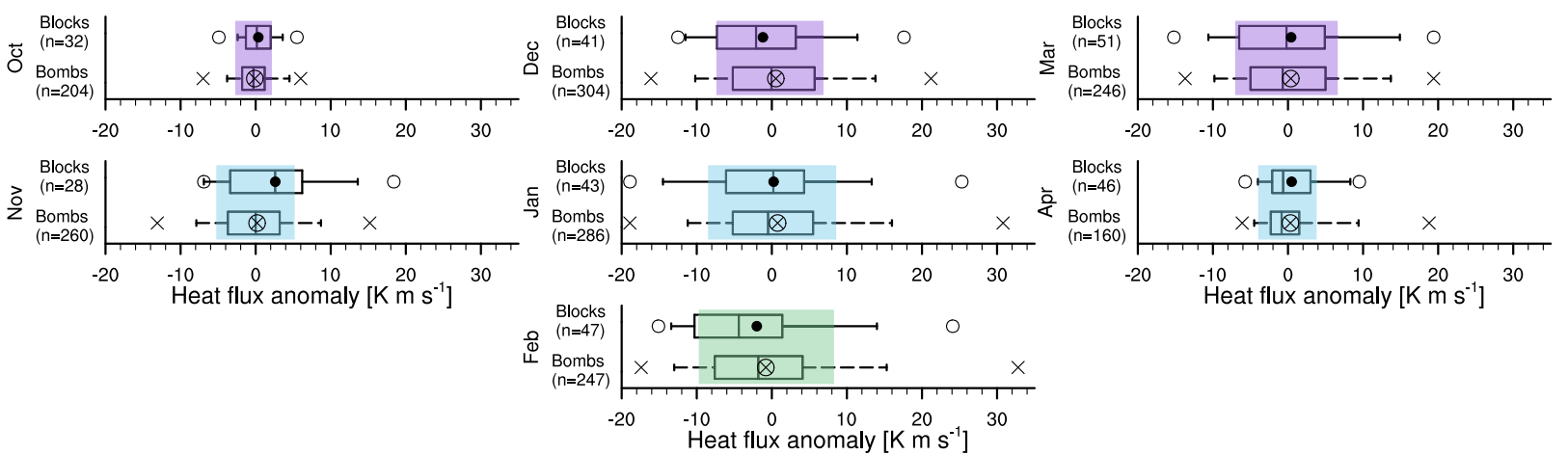

FIG. 1. Intraseasonal variation of the 11-day-average heat flux anomaly following all (a) October and November days, (b) DecemberFebruary days, and (c) March and April days. Each panel shows the probability distribution function (PDF) of the 11-day-average 100-hPa heat flux anomaly following all cool-season days (black), all days in each subseason category (tan), and all days in each month (purple, blue, or green) according to the legend in each plot. The dashed lines are for the same periods but for the 11-day-average 250-hPa heat flux anomaly. The box-and-whisker plots of the 11-day-average 100-hPa heat flux anomaly following all blocks (circles and solid whiskers) and all bombs (crosses and dashed whiskers) in each month are below the PDFs. The 25th and 75th percentiles are represented by the left-hand side and right-hand side of the box respectively, the 5 th and 95 th percentiles by the whiskers, the median by the center line, the mean by the block dot, and the maximum and minimum by the open circles (crosses). The shading corresponds to 1 standard deviation of all days in that month included in the study.

November, however, are skewed toward anomalously positive heat flux anomalies, as the 75th percentile, represented by the right-hand side of the box, is outside of the +1 standard deviation threshold of all November days and the maximum falls at the tail of the November PDF (Fig. 1a). For blocks that occur in February, more than $25 \%$ of events have heat flux anomaly less than -1 standard deviation of the mean February heat flux anomaly (Fig. 1b). The distribution of heat flux anomalies for blocks and bombs is similar at $250 \mathrm{hPa}$ (not shown).

\section{b. Variability associated with block and bomb locations}

Examining the heat flux anomaly of blocks by location shows that the interquartile range extends outside of \pm 1 standard deviation for west Pacific (Fig. 2b), European (Fig. 2c), east Pacific (Fig. 2d), and Asian blocks (Fig. 2e). Although the 25th percentile of the heat flux anomaly of Asian blocks falls outside the bounds of -1 standard deviation, the small number of identified blocks in Asia $(n=22)$ suggests that these events are not frequent. For European blocks, the heat flux anomaly distribution is displaced toward positive anomalies (Fig. 2c), while for both west and east Pacific blocks the distribution is displaced toward negative anomalies (Fig. 2d).

Motivated by previous studies that highlighted the importance of blocking in the Euro-Atlantic and west Pacific regions prior to SSWs (e.g., Martius et al. 2009; Nishii et al. 2011), European and west Pacific blocks are analyzed in more detail. Although the mean 100-hPa 11-day heat flux anomaly is positive following European blocks and negative following west Pacific blocks, the variability in the 11-day-average heat flux anomaly following blocks within each region varies greatly. The difference between the maximum and minimum heat flux anomalies for both regions is $30 \mathrm{~K} \mathrm{~m} \mathrm{~s}^{-1}$ (Figs. 2b,c). Consistent with Nishii et al. (2011), the daily average time series of the heat flux anomaly shows that 

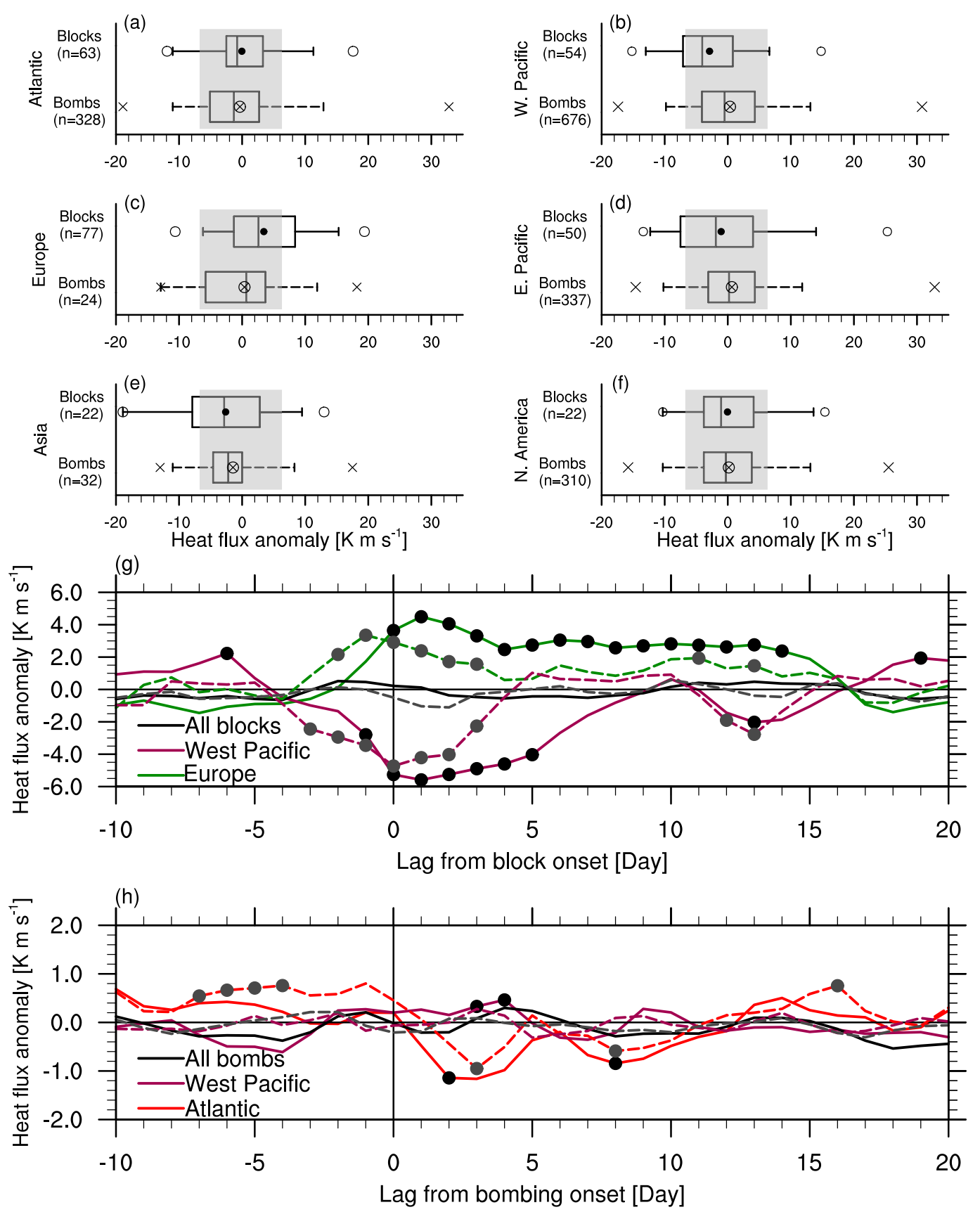

FIG. 2. (a)-(f) As in the box-and-whisker plots in Fig. 1, but for events partitioned by the locations outlined in Table 1. The gray shading indicates one standard deviation of the 11-day-average 100-hPa heat flux anomaly all cool-season days. (g),(h) Time series of the 100-hPa heat flux anomaly (solid) and 250-hPa heat flux anomaly (dashed) following onset of blocks and bombs in the regions indicated by the legends. Dots indicate statistical significance at the $95 \%$ level with respect to the same number of cases in each region randomly selected from ( $\mathrm{g}$ ) all blocks or (h) bombs.

following the onset of European blocks $(n=77)$, there are statistically significant mean positive heat flux anomalies while following the onset of west Pacific blocks $(n=54)$, there are statistically significant mean negative heat flux anomalies (Fig. 2g). At $250 \mathrm{hPa}$, the increase (or decrease) of heat flux anomaly and its statistical significance precedes the onset of the blocks and the period of statistical significance at $100 \mathrm{hPa}$. The $100-\mathrm{hPa}$ heat flux anomaly lagging the $250-\mathrm{hPa}$ heat flux anomaly suggests that a good portion of the 
TABLE 2. The number of bombs in the top (T25) and bottom (B25) quartiles of all Atlantic and west Pacific bombs at both 100 and $250 \mathrm{hPa}$ based on the standardized heat flux anomaly.

\begin{tabular}{ccc}
\hline & Atlantic $(n=82)$ & West Pacific $(n=169)$ \\
\hline T25 & $51(62.2 \%)$ & $101(59.8 \%)$ \\
B25 & $44(53.7 \%)$ & $97(57.4 \%)$ \\
\hline
\end{tabular}

$100-\mathrm{hPa}$ heat flux anomaly that follows blocks originated near the tropopause.

The interquartile range of heat flux anomaly following bombs in each location falls within \pm 1 standard deviation of the mean of all cool-season days (Figs. 2a-f). The heat flux anomaly distribution for west Pacific bombs is slightly skewed toward positive heat flux anomalies (Fig. 2b). While the middle $50 \%$ of heat flux anomalies following Atlantic bombs is well within \pm 1 standard deviation, the maximum and minimum heat flux anomalies following Atlantic bombs represent the maximum and minimum of all bombs in the dataset (Fig. 2a).

Motivated by Coy and Pawson (2015), who showed the importance of a rapidly deepening Atlantic extratropical cyclone prior to the January 2013 SSW and the statistically significant mean positive heat flux anomalies following west Pacific bombs shown in Fig. 2h, bombs that occurred in the Atlantic and west Pacific regions are analyzed in more detail. The mean heat flux anomalies in the days following the onset of Atlantic bombs $(n=328)$ and west Pacific bombs $(n=676)$ are negative and positive, respectively (Fig. 2h), thus is opposite signed to that following blocks in the same hemisphere (Fig. $2 \mathrm{~g}, \mathrm{~h}$ ). At $250 \mathrm{hPa}$, only Atlantic bombs have statistically significant heat flux anomaly (Fig. 2h). Around a week prior to onset of Atlantic bombs there is statistically significant positive heat flux anomaly that becomes negative and is statistically significant 3 days after onset. The significant heat flux anomaly at $250 \mathrm{hPa}$ does not precede the significant heat flux anomaly at $100 \mathrm{hPa}$ like was seen for blocks (Figs. 2g,h). However, the evolution of the heat flux anomaly from positive to negative surrounding the onset of Atlantic bombs suggests that the tropopause wave train may play an important role in the sign of the heat flux anomaly, such that as the tropopause wave packet associated with the bombs presumably progresses eastward across the Atlantic, the sign of the heat flux anomaly reverses.

\section{c. Extreme heat flux anomalies}

To examine the synoptic events in the regions of interest followed by the most extreme heat flux anomalies, events were listed according to their 11-day-average $100-\mathrm{hPa}$ heat flux anomaly, and the blocks and bombs
TABLE 3. As in Table 2, but for European and the west Pacific blocks.

\begin{tabular}{ccc}
\hline & Europe $(n=19)$ & West Pacific $(n=14)$ \\
\hline T25 & $10(52.6 \%)$ & $8(57.1 \%)$ \\
B25 & $10(52.6 \%)$ & $7(50.0 \%)$ \\
\hline
\end{tabular}

representing the top and bottom $25 \%$ of heat flux anomalies, hereafter T25 and B25, respectively, were analyzed in more detail.

Corresponding lists were also created based on the heat flux anomaly at $250 \mathrm{hPa}$ as well as lists from the standardized heat flux anomalies at 100 and $250 \mathrm{hPa}$. Tables 2 and 3 show the number of events that overlap between the $100-$ and $250-\mathrm{hPa}$ standardized heat flux anomaly lists. At least $50 \%$ of the events in each category where identified on both lists, with almost all of the bomb categories having at least $60 \%$ of the events on each list (Table 2). The consistency between lists suggests that the majority of synoptic cases with extreme heat flux anomaly at the tropopause level also have extreme heat flux at $100 \mathrm{hPa}$, supporting the idea that in the majority of cases, the heat flux anomaly is generally of tropospheric origin.

To put the synoptic event heat flux anomalies into context of the full spectrum of cool-season heat flux events, lists of extreme cool-season heat flux events were created. Unique heat flux events were identified by listing the 11-day-average 100-hPa heat flux anomaly following all cool-season days included in this study $(n=7428)$ in descending order and retaining the top and bottom $10 \%(n=743)$. Any event in the top and bottom $10 \%$ that occurred within 10 days of a date with a greater magnitude of 11-day-average heat flux anomaly was removed from consideration. The final list corresponds to 84 unique top $10 \%$ heat flux events and 95 unique bottom $10 \%$ heat flux events, hereafter top heat flux events and bottom heat flux events, respectively. Following the same methodology, lists of unique extreme heat flux events based on the $100-\mathrm{hPa}$ standardized anomaly were also created and corresponds to 95 top events and 106 bottom events. The unique 100-hPa heat flux anomaly lists are cross referenced with the T25 and B25100-hPa anomaly events while the unique $100-\mathrm{hPa}$ heat flux standardized anomaly lists are cross referenced with the $\mathrm{T} 25$ and $\mathrm{B} 25250-\mathrm{hPa}$ standardized anomaly events. When one of the T25 or B25 synoptic events occurred within \pm 5 days of a top or bottom heat flux event, the two events were said to be associated.

Of the 84 top $100-\mathrm{hPa}$ heat flux anomaly events, 78 $(93 \%)$ were associated with a T25 $(n=284)$ or B25 $(n=284) 100-\mathrm{hPa}$ heat flux anomaly block or bomb and 
(a)

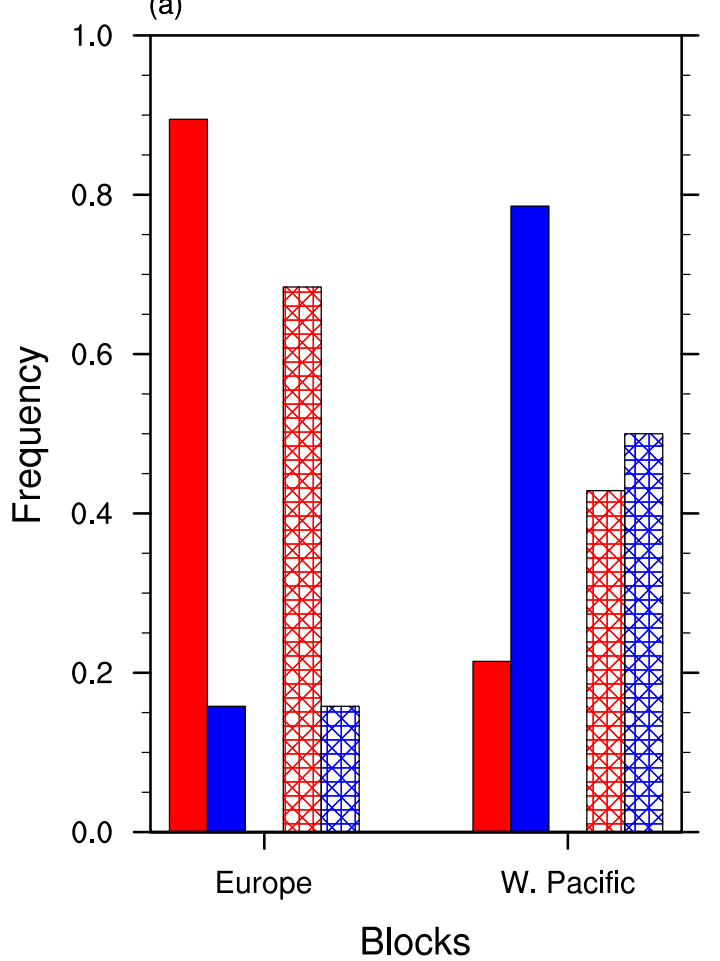

(b)

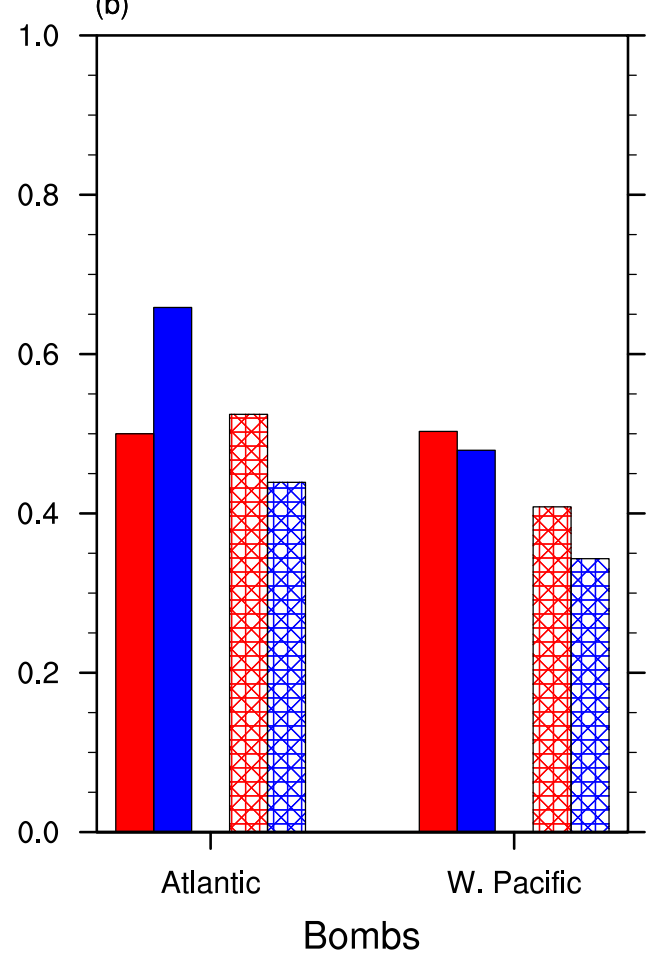

FIG. 3. The frequency of 100-hPa T25 (solid red) and B25 (solid blue) synoptic events that occur within \pm 5 days of all 100-hPa top $(n=84)$ and bottom $(n=95)$ heat flux events and the frequency of the 250-hPa standardized anomaly T25 (patterned red) and B25 (patterned blue) synoptic events that occur within \pm 5 days of all 100-hPa standardized anomaly top $(n=95)$ and bottom $(n=106)$ heat flux events. Frequencies are with respect to the number of T25 or B25 events in each region (i.e., 19 European blocks, 14 west Pacific blocks, 82 Atlantic bombs, and 169 west Pacific bombs).

of the 95 bottom heat flux events, 83 (87\%) were associated with a T25 or B25 block or bomb. This relationship is slightly weaker but still strong when considering the 95 unique top and 106 unique bottom $100-\mathrm{hPa}$ standardized anomaly heat flux events and their relationship with the T25 and B25250-hPa standardized anomaly events. Seventy-six $(80 \%)$ of the unique top $100-\mathrm{hPa}$ standardize anomaly events were associated with a T25 or B25 250-hPa event and 67 (63\%) unique bottom events were associated with a T25 or B25250-hPa event. These associations between the top and bottom heat flux events and the T25 and B25 blocks and bombs are statistically significant at the $5 \%$ level with respect to bootstrap resampling of all cool-season days for all categories except the $67100-\mathrm{hPa}$ standardized anomaly unique bottom events.

With respect to the 100-hPa heat flux anomaly, almost all T25 European blocks and only $50 \%$ of the T25 west Pacific bombs fall within the top heat flux events of all cool-season days (Fig. 3). Conversely, most B25 west Pacific blocks and over half of the B25 Atlantic bombs fall in the bottom heat flux events of all cool-season days (Fig. 3). This suggests that while the range of heat flux anomalies following synoptic events in the EuroAtlantic and Pacific regions may be similar (i.e., around $30 \mathrm{~K} \mathrm{~m} \mathrm{~s}^{-1}$ ), where events in these regions fall with respect to the most extreme anomalies is not necessarily the same. The distributions of the $250-\mathrm{hPa}$ standardized heat flux anomaly T25 and B25 events are similar to the distributions of the $100-\mathrm{hPa}$ heat flux anomaly T25 and B25 events, though of a smaller magnitude (Fig. 3). There is a notable difference for T25 and B25 250-hPa Atlantic bombs, such that a higher percentage of $\mathrm{T} 25$ events were associated with a unique top 100-hPa day than B25 events. The association between the synoptic events and top heat flux events suggests that the dynamics of synoptic events should be important considerations when analyzing extreme heat flux events.

\section{Temporal analysis of heat flux anomaly surrounding blocks and extratropical cyclones}

Given that the majority of our cases are preceded by a similarly signed heat flux anomaly extreme at 250 and $100 \mathrm{hPa}$ and that $100 \mathrm{hPa}$ is primarily the focus of heat 
flux analyses in the literature, the following sections focus on analysis of the 100-hPa heat flux anomalies.

\section{a. European and west Pacific blocks}

The daily averaged mean heat flux anomaly surrounding onset of T25 and B25 European blocks are statistically different from each other from days -1 to +15 (Fig. 4a). The heat flux anomaly decomposition terms for the T25 and B25 European blocks are all statistically different from days +2 to +10 , indicating that each term is playing a significant role in contributing to their respective total heat flux anomaly (Fig. 4a). The terms with the anomalous meridional wind (i.e., $\left[v_{a}^{*} T_{c}^{*}\right]$ and $\left[v_{a}^{*} T_{a}^{*}\right]_{a}$ ) are the largest contributors to the T25 total heat flux anomaly suggesting that the constructive interaction of the anomalous meridional wind with the full (i.e., both the climatological and anomalous) temperature field is an important feature of European blocks that are followed by anomalously positive heat flux (Fig. 4a). Conversely, for the B25 European blocks, the two terms with the anomalous temperature (i.e., $\left[v_{c}^{*} T_{a}^{*}\right]$ and $\left[v_{a}^{*} T_{a}^{*}\right]_{a}$ ) contribute the most to the total negative heat flux anomaly (Fig. 4a). This suggests that the interaction between the anomalous temperature field with the full meridional wind field is an important driver of negative heat flux anomaly following European blocks. This difference in the decomposition terms that dominate the T25 and B25 total heat flux anomaly suggests that for the European blocks, the T25 total heat flux anomaly can be largely attributed to anomalous perturbations to the momentum field, but for the B25 events, the total heat flux anomaly can be largely attributed to anomalous perturbations to the thermal field.

The analysis also shows that there is variability in the seasonality of the T25 and B25 European blocks. The B25 European blocks occur more frequently in the spring (i.e., March and April) while the T25 European blocks occur primarily from December to March (Fig. 4b). No European blocks followed by extreme heat flux anomalies occurred in October, which is statistically significant with respect to what is expected from all European blocks (Fig. 4b).

The daily averaged total heat flux anomaly surrounding the onset of the T25 and B25 west Pacific blocks shows significant differences from day -1 to +11 (Fig. 4c). For the B25 west Pacific blocks, the heat flux anomaly begins to decrease before blocking onset, but the T25 heat flux anomaly does not increase until day +3 and reaches its peak on day +8 , before decreasing to near climatological heat flux by day +12 (Fig. 4c). The $\mathrm{T} 25\left[v_{a}^{*} T_{a}^{*}\right]_{a}$ term follows a similar evolution to the T25 total heat flux anomaly. The B25 $\left[v_{a}^{*} T_{a}^{*}\right]_{a}$ term remains

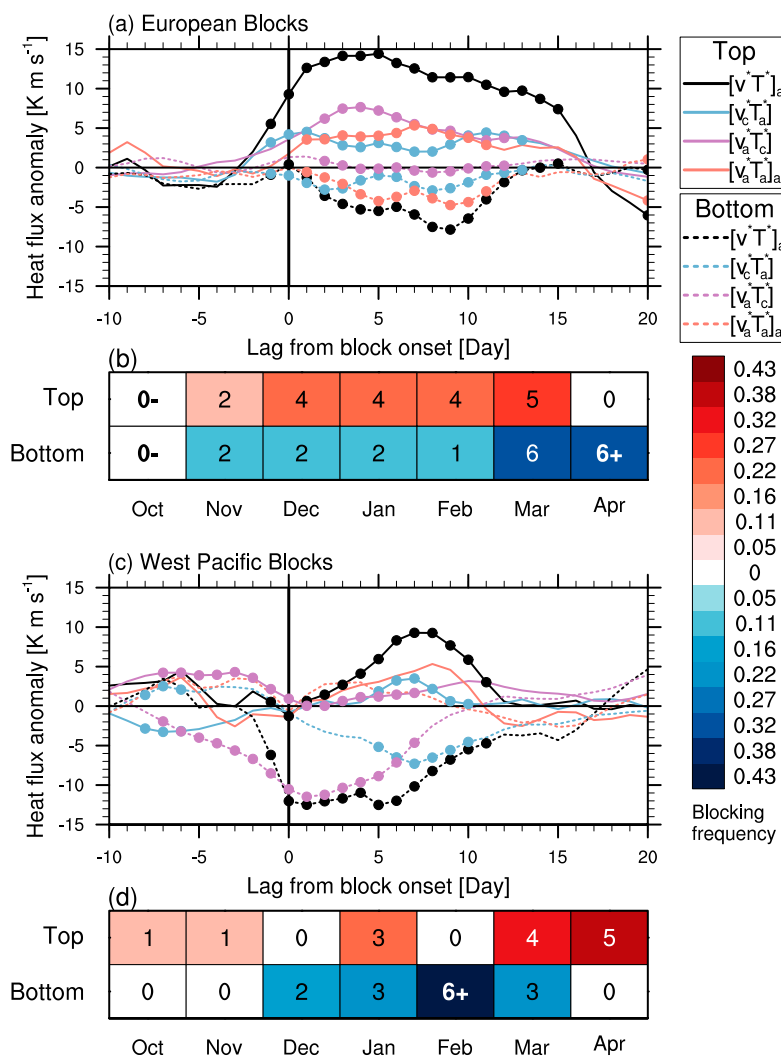

FIG. 4. Time series of the decomposition of the heat flux anomalies [right hand side of Eq. (1); terms colored according to the legend] for the T25 group (solid lines) and the bottom B25 group (dashed lines) of (a) European blocks and (c) west Pacific blocks. (b),(d) Heat plots of the intraseasonal variation of the frequency of the $\mathrm{T} 25$ and B25 events by month in each region.

positive until day +8 and the $\mathrm{T} 25$ and $\mathrm{B} 25\left[v_{a}^{*} T_{a}^{*}\right]_{a}$ terms are not statistically different from each other through the 30-day period analyzed (Fig. 4c).

For the B25 west Pacific blocks, the interaction terms, $\left[v_{a}^{*} T_{c}^{*}\right]$ and $\left[v_{c}^{*} T_{a}^{*}\right]$ are the main contributors to the total negative heat flux anomaly (Fig. 4c). The different temporal evolutions of the $\mathrm{B} 25\left[v_{a}^{*} T_{c}^{*}\right]$ and $\left[v_{c}^{*} T_{a}^{*}\right]$ terms suggests that the interaction of the climatological temperature field with the anomalous meridional wind field (i.e., the $\left[v_{a}^{*} T_{c}^{*}\right]$ term) is the main contributor to the negative heat flux anomaly in the week leading to the B25 block onset and maintains the negative heat flux anomaly for multiple days after B25 block onset (Fig. 4c). The B25 [ $\left.v_{a}^{*} T_{c}^{*}\right]$ term is statistically different from its T25 counterpart up to a week prior to block onset. Overall, the analysis suggests that the interaction between the anomalous meridional and anomalous thermal fields will most likely contribute positively to the total heat flux anomaly following west Pacific blocks, whether the total heat flux anomaly is positive or negative. The interaction of these anomalies with 
the climatological flow, however, determines the sign of the total heat flux anomaly.

The monthly distribution of west Pacific blocks followed by extreme heat flux anomalies is quite different from that of the European blocks (Figs. 4b,d). The statistically significant peak month of occurrence for B25 west Pacific blocks was February, with all blocks in the B25 group occurring from December to March (Fig. 4d). The T25 west Pacific blocks, however, had its peak in April and no events in December or February.

The analysis of the heat flux anomaly following blocks suggests that anomalies of certain signs have preferred locations and time of year of occurrence. European blocks in the middle of the cool season are likely followed by anomalously positive heat flux, but west Pacific blocks during these same periods are likely followed by anomalously negative heat flux.

\section{b. Atlantic and west Pacific bombs}

The T25 and B25 heat flux anomalies for Atlantic bombs are statistically different from day -4 to day +13 with all three T25 decomposition terms contributing similar magnitudes to the total T25 heat flux anomaly (Fig. 5a). For the B25 group, the two interaction terms have magnitudes that are comparable to each other and larger than the $\left[v_{a}^{*} T_{a}^{*}\right]_{a}$ term, which has a magnitude that is negative but small for the entire period (Fig. 5a). The intraseasonal distribution of Atlantic bombs associated with the most extreme heat flux anomalies shows that the B25 events have statistically significant maxima in January and February and statistically significant minima in October and April (Fig. 5b). The T25 Atlantic bombs, however, do not have a clear intraseasonal evolution as there is a statistically significant frequency maximum in March with a secondary maximum in January (Fig. 5b). Similar to the B25 events, there is also a statistically significant T25 frequency minimum in October.

For west Pacific bombs, each decomposition term is statistically different between the T25 and B25 groups from day -3 to at least day +11 ; the $\left[v_{c}^{*} T_{a}^{*}\right]$ terms are statistically different until day +20 (Fig. 5c). The three decomposition terms of the T25 west Pacific bombs contribute similar magnitudes to the total heat flux anomaly, much like the T25 Atlantic bombs (Figs. 5a,c). The three decomposition terms for the B25 west Pacific bombs are also of comparable magnitudes to each other (Fig. 5c), which is in contrast to the total B25 Atlantic heat flux anomaly, which was dominated by the interaction terms (Fig. 5a). The similar magnitudes of the three decomposition terms for the B25 west Pacific bombs suggests that the phasing of the anomalous meridional wind and thermal fields play a more active role

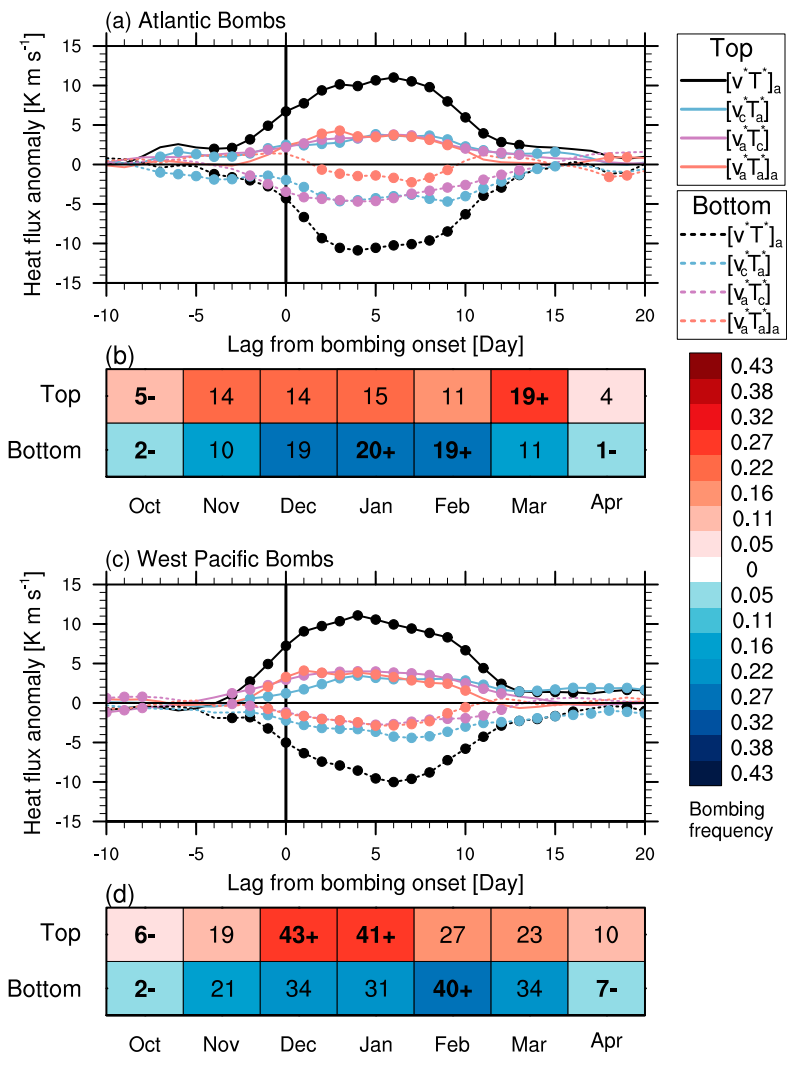

FIG. 5. As in Fig. 4, but for bombing in the (a),(b) Atlantic and (c),(d) west Pacific.

in leading to negative heat flux anomaly following west Pacific bombs than following Atlantic bombs.

The intraseasonal variability of the T25 west Pacific bombs shows statistically significant maxima in December and January (Fig. 5d). The B25 west Pacific bombs have a statistically significant peak frequency in February with secondary maxima in December and March (Fig. 5d). Both the T25 and B25 west Pacific bombs have frequency minima during October that are statistically significant with respect to all west Pacific bombs.

\section{c. Discussion}

The analysis shows that blocks and bombs associated with extreme heat flux anomalies do not necessarily occur during the same time of year. In the Euro-Atlantic sector, blocks followed by negative heat flux anomaly are more frequent in March and April, while bombs followed by negative heat flux anomaly occur more frequently in January and February (Figs. 4b and 5b). In the west Pacific, blocks associated with positive heat flux anomaly occur more often in March and April, but west Pacific bombs associated with positive heat flux anomalies more likely earlier in the winter: December and January (Figs. 4d and 5d). All categories except T25 
and B25 west Pacific blocks had statistically significant frequency minima during October. This suggests that if a European block, Atlantic bomb, or west Pacific bomb occurs in October it will likely not be associated with extreme heat flux anomalies.

To explore the evolution of the planetary-scale pattern at $100 \mathrm{hPa}$, where this vertical WAF is (or is not) occurring, the evolution of the $60^{\circ} \mathrm{N}$ geopotential height waves is analyzed. Figure 6 shows that, on average, blocks and bombs associated with anomalously positive heat flux are also associated with increasing wavenumber 1 (WN1) and wavenumber 2 (WN2) amplitudes with time. The exception to this is T25 west Pacific blocks, which had a WN1 maximum amplitude that decreased with time. Blocks and bombs followed by negative heat flux anomalies, however, are associated with decreasing WN1 and WN2 amplitudes with time (Fig. 6). This analysis suggests that diagnosing how the planetary-scale environment evolves could be an indication of the sign of heat flux anomaly. To further explore the horizontal and vertical structure of the atmosphere surrounding event onset, the following section will analyze the structure of the geopotential height.

\section{Spatial analysis}

\section{a. Geopotential height}

At block onset, the T25 European blocks are associated with a broad tropospheric ridge that is baroclinically phased with the stratospheric WN1 structure, exemplified by the westward tilt with height (Fig. 7a). The differences between the T25 and B25 groups are statistically significant in the troposphere (i.e., 1000$200 \mathrm{hPa}$ ) in the vicinity of the composite blocking location, around $0^{\circ}$, as well as downstream of the block, from $60^{\circ}$ to $120^{\circ} \mathrm{E}$ near the surface (Fig. 7c). The T25 European blocks have larger stratospheric geopotential height anomaly maxima than the B25 European blocks (Figs. 7a,b). These differences are statistically significant between $180^{\circ}$ and $60^{\circ} \mathrm{E}$ above $\sim 50 \mathrm{hPa}$ (Fig. $7 \mathrm{c}$ ). The vertical cross sections suggest that European blocks that are followed by positive heat flux anomalies are not only more robust in terms of the their midtropospheric amplitude, but also have a more robust Siberian surface high and a more robust and eastward displaced mid- to upper-stratospheric Aleutian high (i.e., $10-1 \mathrm{hPa}$ ). These robust $\mathrm{T} 25$ stratospheric height anomalies correlate to an amplified maximum WN1 magnitude from the upper troposphere to the stratosphere and a robust and eastward displaced $10-\mathrm{hPa}$ Aleutian high and displaced polar vortex (Fig. 8a). (a)

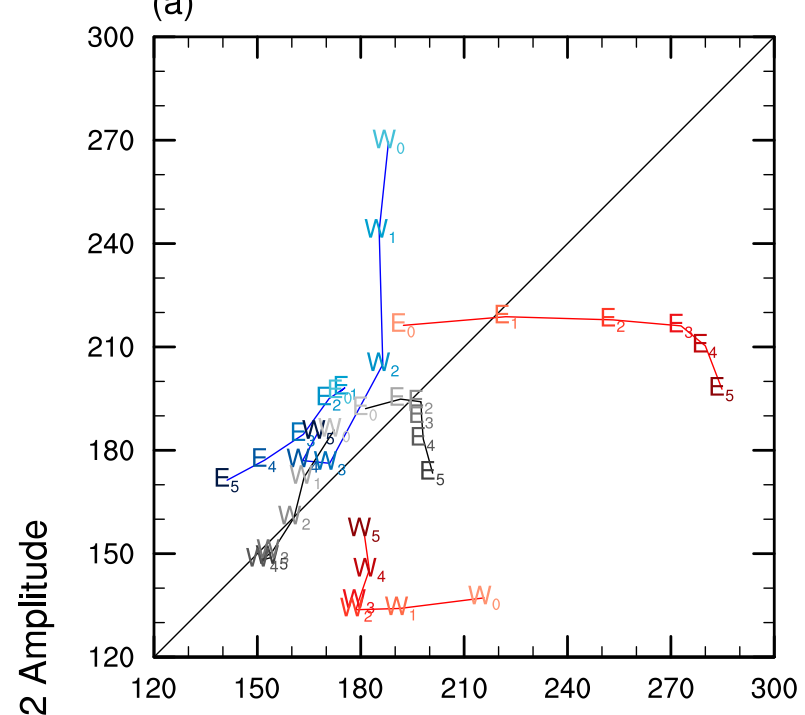

(b)

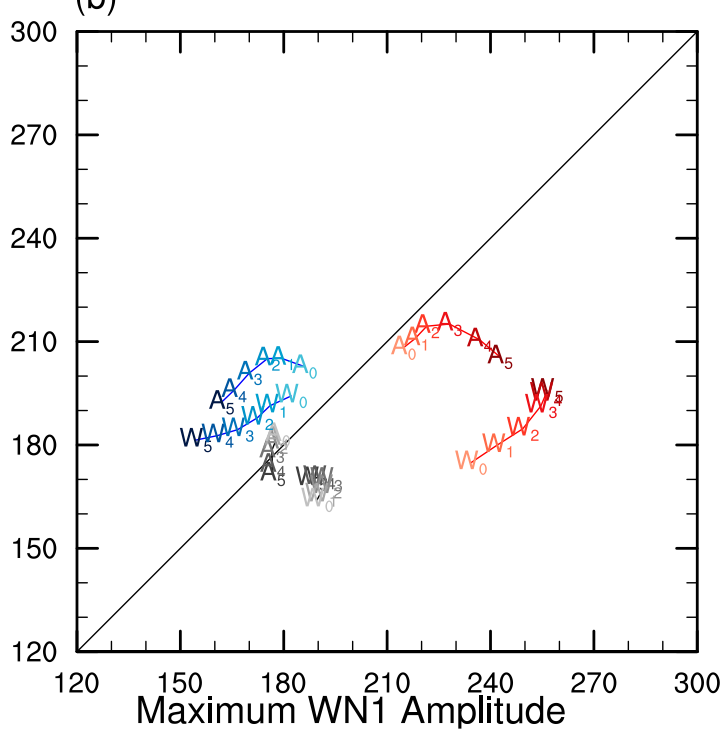

FIG. 6. Phase space of the $100-\mathrm{hPa} 60^{\circ} \mathrm{N}$ maximum WN1 amplitude ( $x$ axis) and WN2 amplitude ( $y$ axis) from lag day 0 to +5 for (a) European (E) and west Pacific (W) blocks and (b) Atlantic (A) and west Pacific (W) bombs in the T25 (red) group, B25 (blue) group, and all events (gray). Subscripts denote the lag day and the colors get darker with increasing day. The diagonal line indicates a 1:1 ratio of WN1:WN2 maximum amplitude.

The west Pacific blocks show a different signal in the vertical structure of the geopotential height anomalies than the European blocks. From the surface to $5 \mathrm{hPa}$, the T25 and B25 west Pacific blocks have statistically significant geopotential height differences in the vicinity of the composite blocking location (Fig. 7f). The barotropic nature of the height difference field highlights key dynamical differences between T25 and B25 west Pacific blocks. The B25 west Pacific blocks have a nearly 
(a) $\mathrm{T} 25$

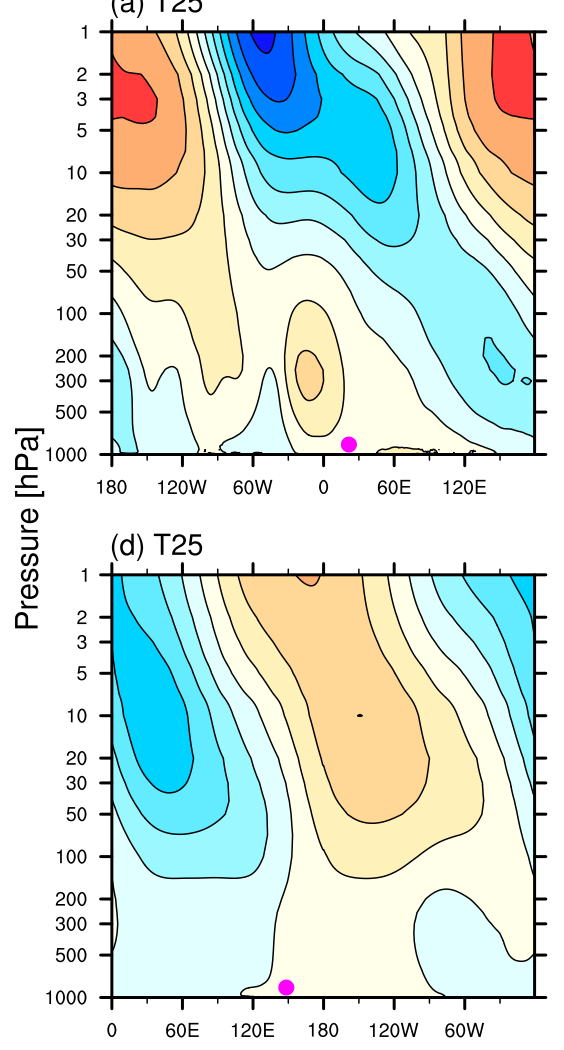

(b) B25

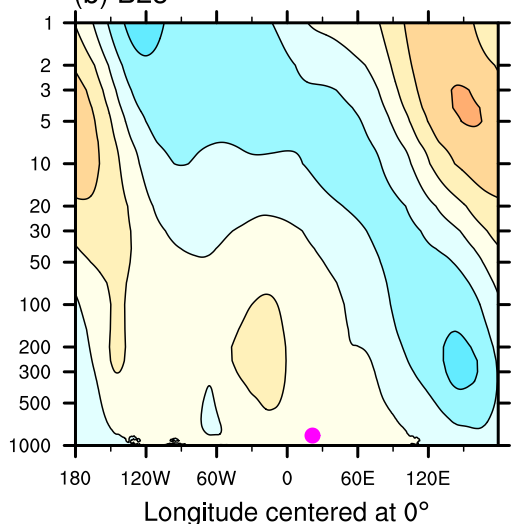

(e) B25

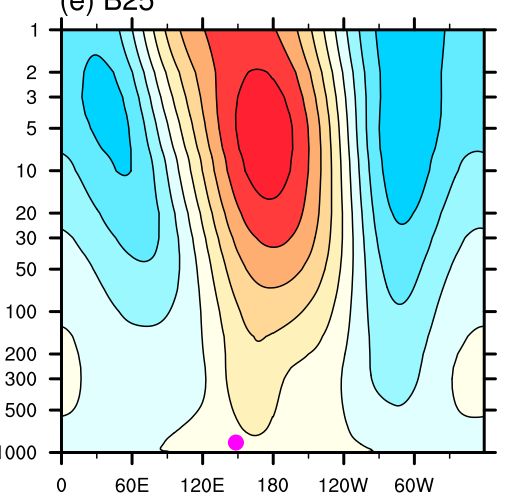

Longitude centered at $180^{\circ}$

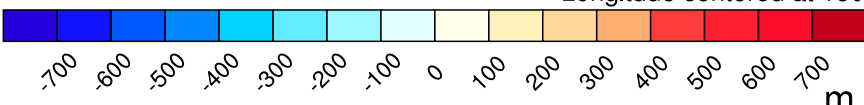

(c) T25 - B25
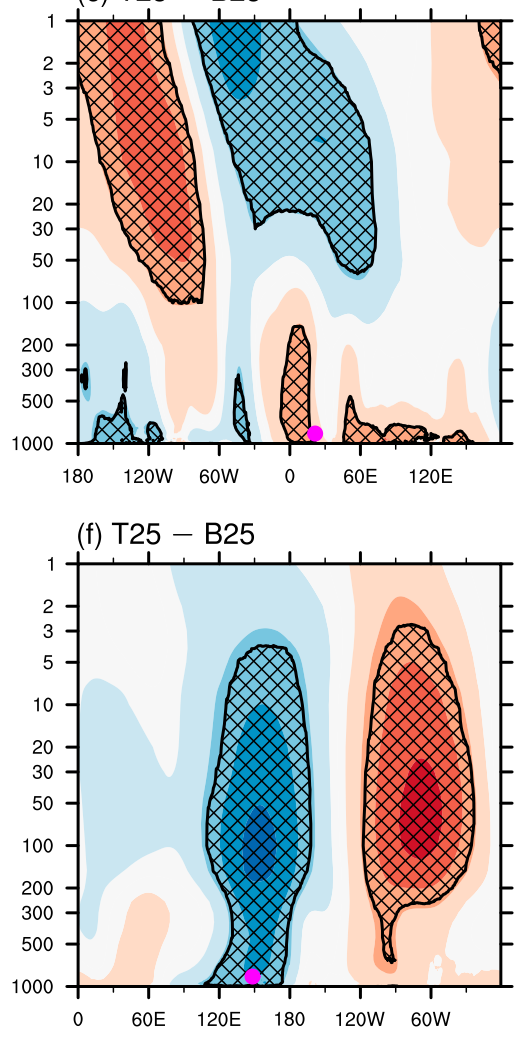

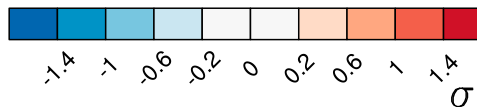

FIG. 7. Event-centered composite analysis of the geopotential height anomalies with respect to the zonal mean averaged between $-5^{\circ} \mathrm{S}$ and $+15^{\circ} \mathrm{N}$ of the composite location of $60^{\circ} \mathrm{N}$ at day 0 for (a)-(c) European blocks and (d)-(f) west Pacific blocks. (a),(d) T25 groups, (b),(e) B25 groups, and (c),(e) normalized differences of geopotential height anomalies between the T25 and B25 groups [i.e., (a) - (b) and (d) $-(\mathrm{e})$, respectively] and statistical significance at the $95 \%$ confidence interval (cross hatching).

barotropic planetary-scale structure, with vertically stacked height anomalies from the surface to $1 \mathrm{hPa}$, suggesting amplified WN1 and WN2 (Fig. 7e). The T25 blocks, however, have a less amplified baroclinic WN1 structure and westward tilted geopotential height anomalies from 100 to $1 \mathrm{hPa}$ (Fig. 7d). At $100 \mathrm{hPa}$, the $\mathrm{B} 25 \mathrm{WN} 2$ field dominates over the WN1 field, while for the T25 group, the $100-\mathrm{hPa}$ WN1 field dominates over the WN2 field (Fig. 6a). These differences between the T25 and B25 groups are associated with an elongated $10-\mathrm{hPa}$ polar vortex that is centered on the polar region in the B25 composite and a circular polar vortex that is displaced toward $30^{\circ} \mathrm{E}$ in the T25 composite (Figs. $8 \mathrm{c}, \mathrm{d}$ ).

The cross section through the T25 and B25 Atlantic bomb locations shows that there are positive tropospheric height anomalies downstream of both Atlantic bomb groups that are statistically different near $60^{\circ} \mathrm{E}$ (Figs. 9a-c). There are also statistical differences in the lower troposphere in the upstream trough between $120^{\circ}$ and $90^{\circ} \mathrm{W}$ (Fig. 9c). The Eastern Hemisphere tropospheric ridge-trough couplet in the T25 bomb composite is associated with cohesive geopotential height anomalies, an amplified baroclinic wave structure with height up to $1 \mathrm{hPa}$ (Figs. 9a-c), and a larger 100-hPa WN1 maximum amplitude than WN2 maximum amplitude (Fig. 6b). The B25 Atlantic bombs exhibit a nearly vertically stacked trough from the bomb location into the lower stratosphere, resulting in a barotropic structure in the vicinity of the composite bomb location (Fig. 9b) and a larger 100-hPa WN2 maximum amplitude than WN1 maximum amplitude (Fig. 6b). The stratospheric geopotential height differences are associated with a more amplified T25 10-hPa Aleutian high and displaced polar vortex toward $40^{\circ} \mathrm{E}$ than the $\mathrm{B} 25$ group, which had a weaker Aleutian high and a vortex centered close to the pole (Figs. 10a,b). The statistical differences between the 
T25

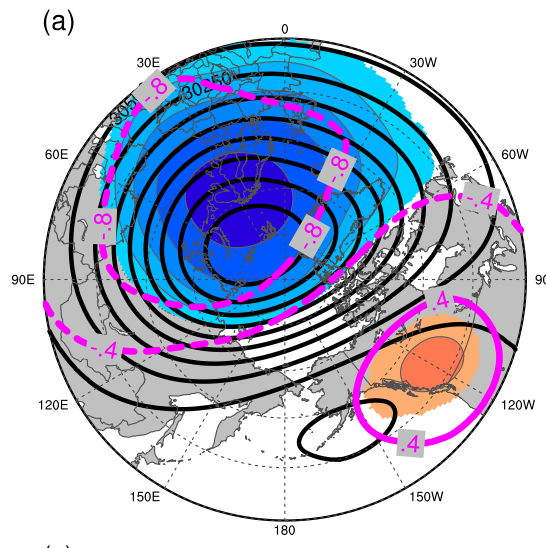

(c)

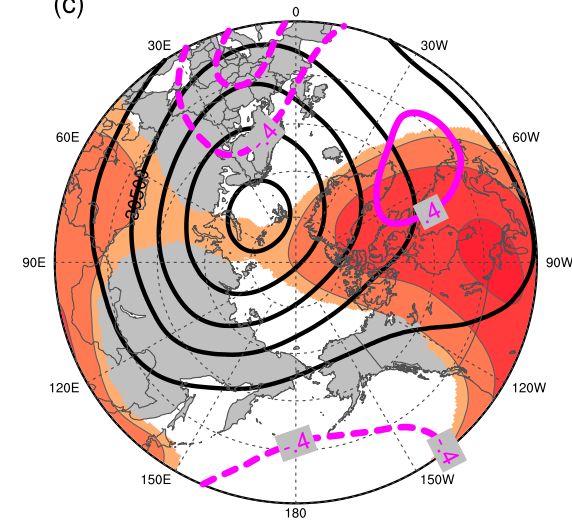

(d)

B25
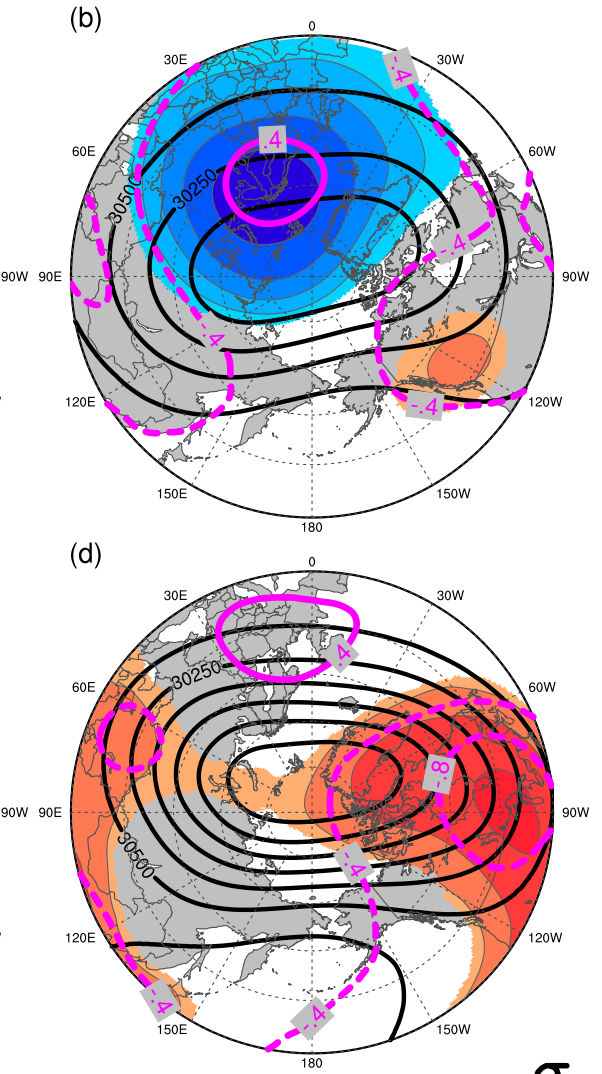

$\sigma$

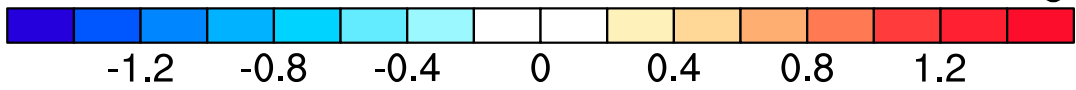

FIG. 8. Non-event-centered Northern Hemisphere composite 10-hPa structure at day 0 with respect to onset of the (left) T25 and (right) B25: (a),(b) European blocks and (c),(d) west Pacific blocks. The 10-hPa geopotential height is contoured every $250 \mathrm{~m}$ (black), the standardized geopotential height anomalies are every $0.4 \sigma$ starting at $0.4(-0.4) \sigma$ [solid (dashed) magenta], and the statistically significant normalized difference between T25 and B25 groups at the $5 \%$ level are shown (shaded).

T25 and B25 Atlantic bombs at the time of bombing from around 200 to $1 \mathrm{hPa}$ (Fig. 9c) suggests that both the planetary-scale stratospheric state and the upper-tropospheric height anomalies downstream of the bomb are important differentiators of bombs with positive and negative heat flux anomalies.

The cross section of the geopotential height anomaly for west Pacific bombs shows that the T25 group has larger magnitudes of anomalies in the stratosphere than the B25 West pacific bomb group (Figs. 9d,e). These larger stratospheric anomalies for the T25 group are associated with a more baroclinic structure within both the troposphere and the stratosphere than the B25 west Pacific composite (Figs. 9d,e). The differences in geopotential height anomaly are maximized in the lower stratosphere but extend throughout the entire atmosphere and highlight the baroclinic nature of the planetary-scale flow that characterizes the T25 group (Figs. 9d-f).

A noticeable difference between the T25 west Pacific bombs and T25 Atlantic bombs is that the T25 west Pacific composite bomb is embedded in low- to midtropospheric negative geopotential height anomalies centered on $180^{\circ}$. This negative anomaly constructively interferes with the stratospheric waves to create a baroclinic environment in the tropopause region, which facilitates wave coupling. In the Atlantic cases, however, the T25 composite bomb is embedded in a broad region of positive tropospheric height anomalies. The Atlantic bomb itself is removed negative tropospheric height anomalies that are centered on $180^{\circ}$ and 
(a) T25

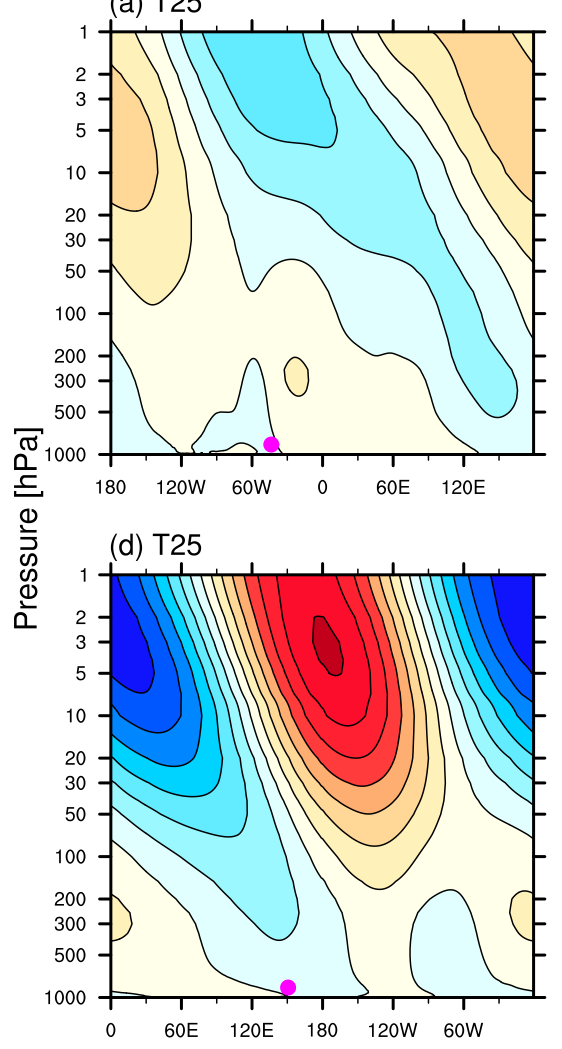

(b) B25

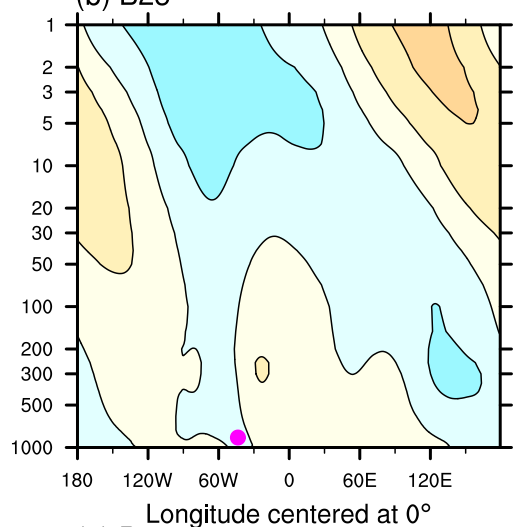

(e) B25

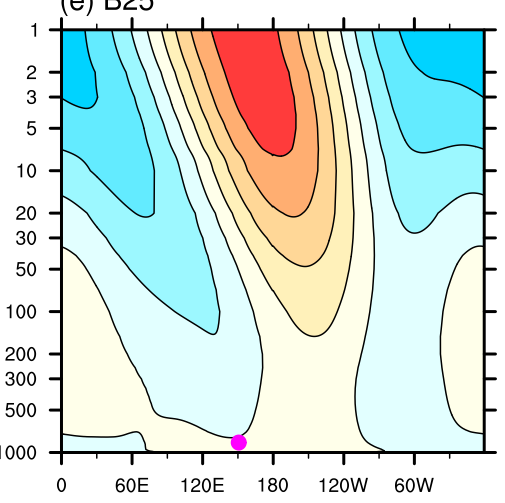

Longitude centered at $180^{\circ}$

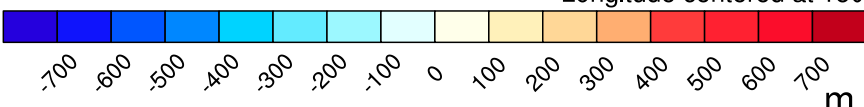

(c) T25 - B25
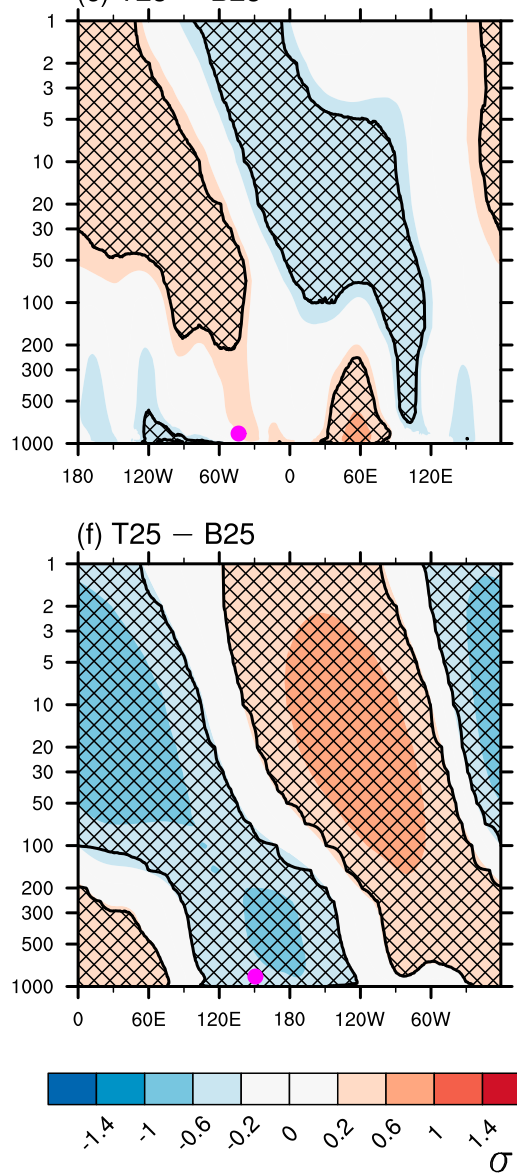

FIG. 9. As in Fig. 7, but averaged between $+10^{\circ}$ and $+30^{\circ} \mathrm{N}$ of the composite location of (a)-(c) $48^{\circ} \mathrm{N}$ for Atlantic bombs and (d)-(f) $40^{\circ} \mathrm{N}$ for west Pacific bombs.

baroclinically phase with the stratospheric negative height anomalies, leading to reduced vertical wave coupling.

\section{b. Heat flux anomaly}

To explore the spatial evolution of the decomposed heat flux anomaly that contributes to the zonal-mean values evaluated in section 4 , nonevent centered composites of the decomposed terms at day +5 are analyzed for the T25 and B25 categories. Day +5 was chosen as it was the day that had the largest difference between the T25 and B25 zonal-mean heat flux anomaly (Figs. 4a,c and $5 \mathrm{a}, \mathrm{c})$. Five days after the onset of European blocking, when the largest differences in the composite zonal-mean heat flux anomaly occurred (Fig. 4a), the T25 $v_{c}^{*} T_{a}^{*}$ and $v_{a}^{*} T_{c}^{*}$ terms are characterized by positive values in Europe and the Pacific (Figs. 11a,c). Over North America, the $v_{c}^{*} T_{a}^{*}$ and $v_{a}^{*} T_{a}^{*}$ terms are negative, which acts to decrease the total zonal-mean heat flux anomaly (Figs. 4a and 11a,g). The T25 and B25 decomposition terms are statistically different over much of the Northern Hemisphere; the B25 decomposition terms have weaker magnitudes than the T25 terms (Fig. 11 right column). The composite shows that European blocks associated with positive heat flux anomaly occur within planetary-scale flow that produces two dominant regions of anomalously positive heat flux maxima: one near the blocking location in Europe and the other in the Pacific (Fig. 11 left column).

At lag day +5 , west Pacific blocks have statistical differences between the T25 and B25 heat flux anomaly decomposition terms (Fig. 12). The B25 magnitudes are larger than the T25 magnitudes (Fig. 12). In the vicinity of blocking location (i.e., the west Pacific), both the T25 and B25 $v_{c}^{*} T_{a}^{*}$ interaction terms are negative (Figs. 12a,b). Although the B25 interaction terms have larger magnitudes in the west Pacific than the T25 interaction terms, statistical differences are mainly confined to North America and Eurasia, where the T25 and B25 terms have opposite signs (Figs. 12a-d). In the B25 cases, the large negative values of the $v_{c}^{*} T_{a}^{*}$ and $v_{a}^{*} T_{c}^{*}$ terms at $180^{\circ}$ and $0^{\circ}$ contribute substantially to the overall negative 
T25
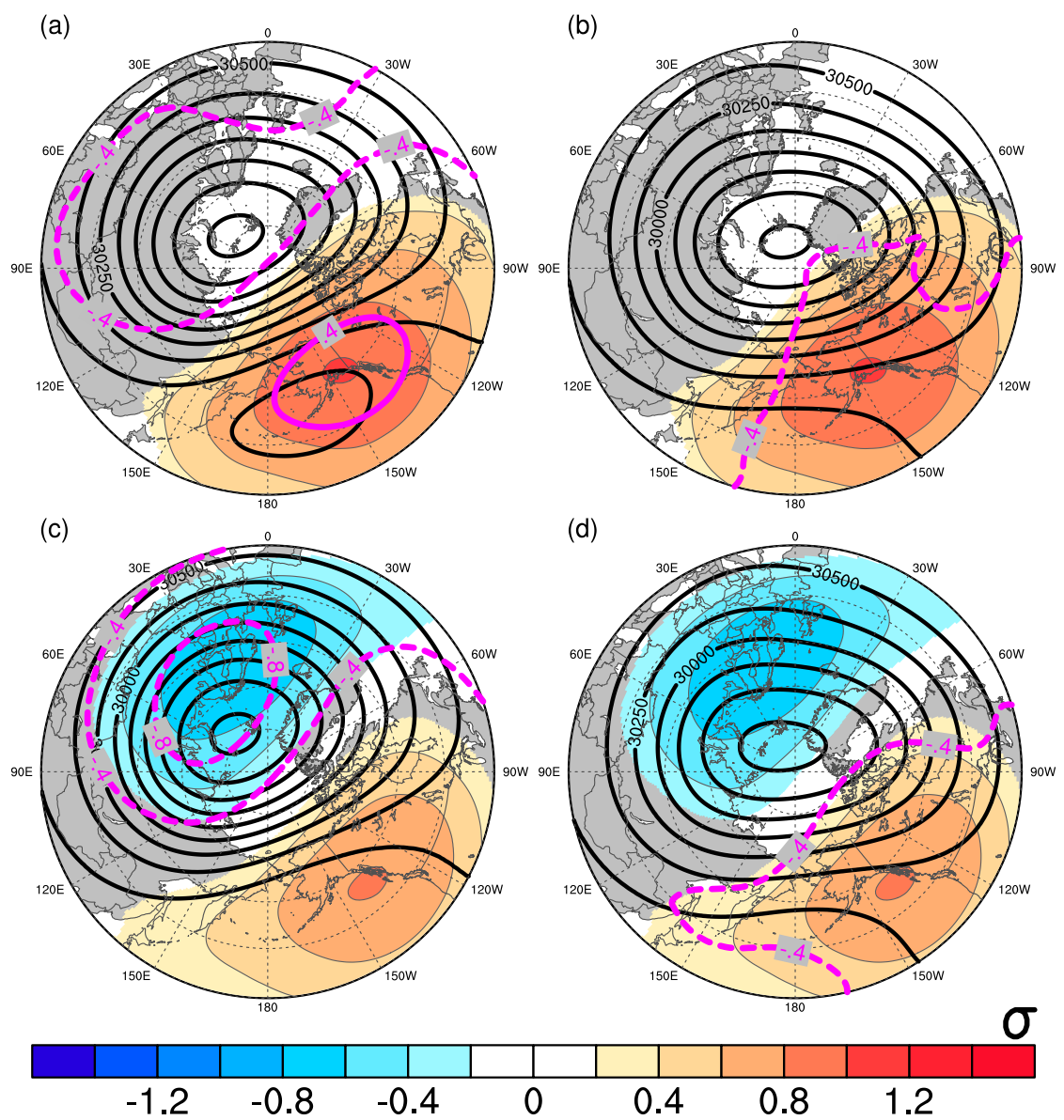

FIG. 10. As in Fig. 8, but for (a),(b) Atlantic bombs and (c),(d) west Pacific bombs. total heat flux anomaly (Figs. 4c and 12f). The spatial distribution of T25 and B25 $v_{a}^{*} T_{a}^{*}$ terms are only statistically different in small regions (Figs. 12g,h). The main difference between the T25 and B25 west Pacific blocks is that for T25 west Pacific blocks, several regions of positive heat flux in the Northern Hemisphere produce the positive total zonal-mean heat flux anomaly (Figs. 12, left column) whereas for the B25 interaction terms, the negative zonal-mean heat flux anomaly comes from negative values of the decomposition terms in two locations: 1) the location of blocking (i.e., the west Pacific) and 2) Eurasia (Figs. 12b,d,f).

The large, but oppositely signed magnitudes of the combined interaction terms from the T25 European blocks and the B25 west Pacific blocks supports the notion that blocks can constructively or destructively interfere with the climatological pattern. In general, European blocks constructively interfere with the climatological pattern to support tropopause heat flux while west Pacific blocks destructively interfere with the climatological pattern and suppress tropopause heat flux, a result supporting Nishii et al. (2011).

On day +5 after onset of Atlantic bombs there are significant spatial differences in the decomposed heat flux anomaly terms between the T25 and B25 groups (Fig. 13), which contribute to large differences in the total zonal-mean heat flux anomaly between these two groups (Fig. 5a). While the T25 $v_{c}^{*} T_{a}^{*}$ and $v_{a}^{*} T_{c}^{*}$ terms are characterized by positive values in the Pacific and Euroasian regions, the B25 Atlantic $v_{c}^{*} T_{a}^{*}$ and $v_{a}^{*} T_{c}^{*}$ terms are negative in those same regions. (Figs. 13a-d). Given that there are relatively small differences in the climatological meridional wind and temperature fields between the T25 and B25 Atlantic bomb composites, the anomalous temperature and meridional wind fields are largely responsible for the opposite signed $v_{c}^{*} T_{a}^{*}$ and $v_{a}^{*} T_{c}^{*}$ terms in the T25 and B25 groups (Figs. 13a-d). The statistically significant spatial difference between the T25 and B25 anomaly terms, $v_{a}^{*} T_{a}^{*}$, are mainly confined to North America (Figs. 13g,h). 
T25
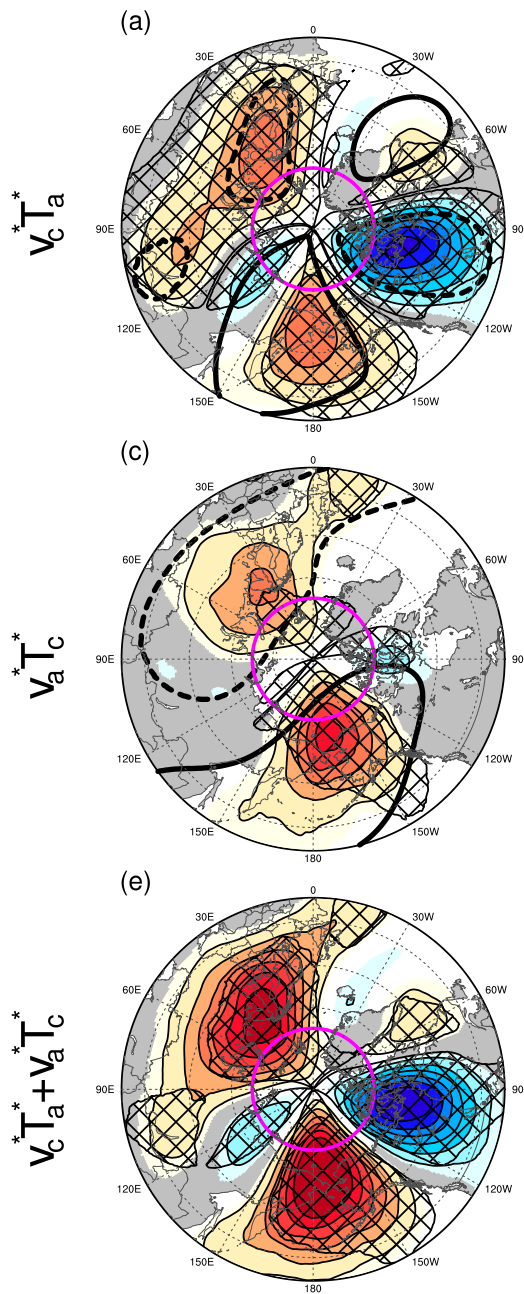

$$
\text { (19) }
$$

(g)

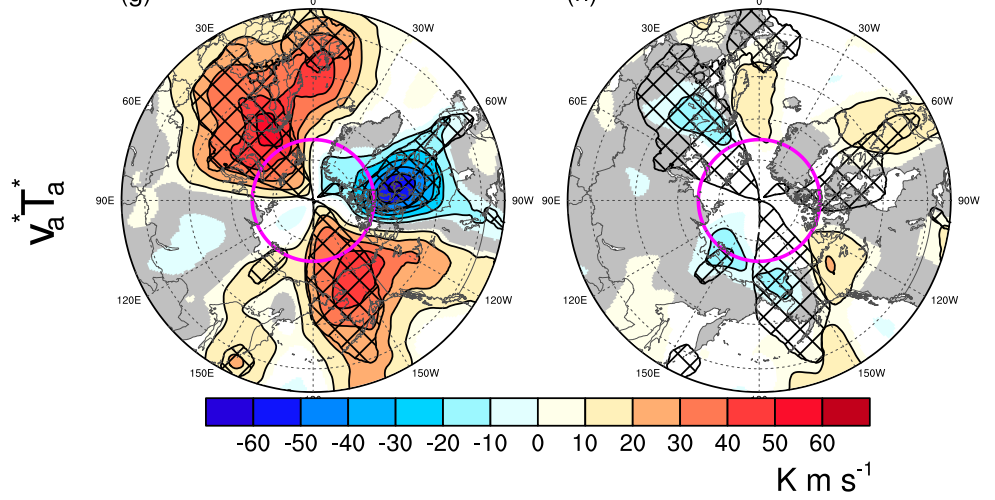

FIG. 11. Non-event-centered composites of the Northern Hemispheric heat flux decomposition for (left) T25 and (right) B25 European blocks. (a),(b) $v_{c}^{*} T_{a}^{*}$ (shaded) and $v_{c}^{*}\left[5(-5) \mathrm{m} \mathrm{s}^{-1}\right.$ in solid (dashed)]; (c),(d) $v_{a}^{*} T_{c}^{*}$ (shaded) and $T_{c}^{*}\left[3(-3) \mathrm{K}\right.$ in solid (dashed)]; (e),(f) $v_{c}^{*} T_{a}^{*}+v_{a}^{*} T_{c}^{*}$ (shaded); (g),(h) $v_{a}^{*} T_{a}^{*}$ (shaded). The cross hatching on each panel indicates regions that are statistically significantly different between the T25 and B25 groups of that variable. The magenta line denotes $75^{\circ} \mathrm{N}$ and the southernmost latitude plotted is $45^{\circ} \mathrm{N}$. 
The T25 and B25 heat flux anomaly decomposition terms for west Pacific bombs are statistically different throughout much of the Northern Hemisphere (Fig. 14). The combined interaction terms show that the T25 west Pacific bombs are characterized by positive values in the Pacific and Eurasian regions and negative values in North America (Fig. 14e), while the B25 combined terms are opposite in those locations (Fig. 14f). The T25 anomalous term is generally positive over much of the Northern Hemisphere, while the B25 anomaly term is small (Figs. 14g,h).

The spatial distribution of the $v_{c}^{*} T_{a}^{*}$ and $v_{a}^{*} T_{c}^{*}$ terms in the T25 west Pacific bombs looks quite similar to the T25 Atlantic bombs, especially in the Pacific (Figs. 13a,c and $14 a, c)$. This similarity suggests that bombs associated with positive zonal-mean heat flux anomalies, regardless of their location, have similar anomalous structures in their temperature and meridional wind fields that constructively interact with the climatological fields to produce positive zonal-mean heat flux anomaly. The $v_{c}^{*} T_{a}^{*}$ B25 Atlantic and west Pacific bombs also have similar spatial distributions to each other, suggesting that bombs with negative zonal-mean heat flux anomaly have similar interactions between the climatological meridional wind and anomalous temperature fields (Figs. 13b and 14b).

\section{c. Conceptual representation of the 100-hPa pattern at day +5}

The planetary-scale patterns of geopotential height, temperature, and meridional wind fields during T25 and B25 European blocks, Atlantic bombs, and west Pacific bombs are consistent across event types and locations. The notable outlier is west Pacific blocks. Figure 15a provides a conceptual diagram of the approximate sign and location of the anomalous and climatological fields of both temperature and meridional wind at $100 \mathrm{hPa}$ representative of the T25 European blocks, Atlantic bombs, and west Pacific bombs at day +5 from the onset of these events. In summary, the T25 cases are characterized by a broad anomalous warm pool in the Western Hemisphere and a broad anomalous cold pool in the Eastern Hemisphere. The anomalous meridional wind field is dominated by a WN1 structure, with poleward winds in the Pacific region and equatorward winds in the EuroAtlantic region associated with a displaced 100 -hPa vortex in the Eastern Hemisphere. Both the anomalous meridional wind and temperature fields represent an enhancement of their respective climatological fields. Other than the anomalous warm pool over North America, which is displaced eastward from the climatological warm pool over North America, the anomalous temperature and meridional wind fields are nearly collocated with their respective climatological fields. Regardless of event type and location, these anomalous meridional and temperature fields interact with the climatological fields in such a way that the combined interaction terms, $v_{c}^{*} T_{a}^{*}$ and $v_{a}^{*} T_{c}^{*}$, are positive in the general Pacific and the Eurasian regions and negative in North America (Figs. 11e, 13e, 14e). The analysis suggests that for any event type and event location to be in the T25 group, the anomalous temperature and meridional wind fields must constructively interact with the climatological meridional wind and temperature fields to induce positive heat flux anomaly on a hemispheric scale and not simply in the local region of the event.

For all the B25 groups, the interaction between the anomalous and climatological fields is opposite-signed to those in the T25 groups with the magnitudes varied between event types (Figs. 11f, 13f, 14f). Figure 15b provides conceptual analysis of the climatological and anomalous temperature and meridional wind fields for the B25 European blocks, Atlantic bombs, and west Pacific bombs. In these events, the anomalous temperature field is characterized by a small cold pool in North America and a small warm pool in Eurasia. The anomalous meridional wind field has weak poleward winds in the Arctic Circle near $0^{\circ}$ longitude and weak equatorward winds in the eastern Pacific. These events are associated with an elongated $100-\mathrm{hPa}$ vortex centered near the pole. These anomalous temperature and meridional wind fields have opposite signs to their collocated climatological fields. This suggests that the anomalous field acts to suppress the climatological fields. Thus, the interaction terms, $v_{c}^{*} T_{a}^{*}$ and $v_{a}^{*} T_{c}^{*}$, in the B25 cases are negative in the Pacific and Eurasia and positive in North America, opposite from the representative T25 cases in Fig. 15a. The synoptic events in the B25 groups are associated with suppressed planetary-scale climatological momentum and thermal fields while the T25 events are associated with enhanced planetary-scale climatological momentum and thermal fields.

The outlier to this conceptual diagram is west Pacific blocks. For the combined interaction terms, the T25 group had negative values in the Pacific and three centers of positive values at: $90^{\circ} \mathrm{E}, 120^{\circ} \mathrm{W}$, and $45^{\circ} \mathrm{W}$ (Fig. 12e). This tripole suggests the potential of higher wavenumbers in west Pacific events with extreme heat flux anomalies. Since both T25 and B25 west Pacific blocks have negative heat flux anomalies in the Pacific, the heat flux anomaly in other locations of the hemisphere must contribute to heat flux extremes in these events. The large magnitude of negative heat flux anomalies in the Pacific and in the western Atlantic in the B25 west Pacific blocks are associated with the $v_{a}^{*} T_{c}^{*}$ term (Fig. 12d), suggesting that the anomalous 
T25

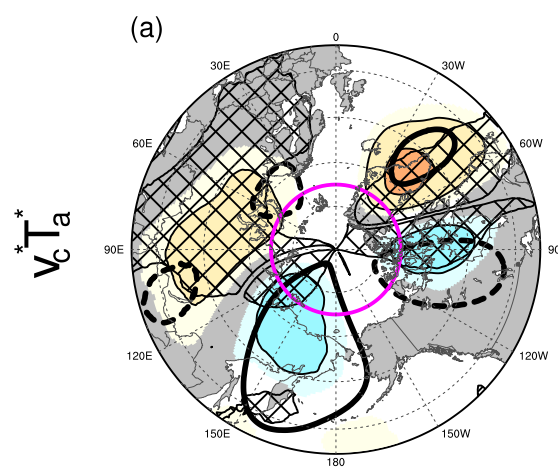

(c)

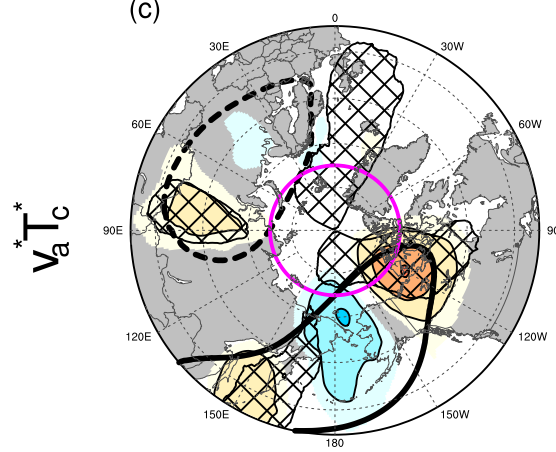

(e)

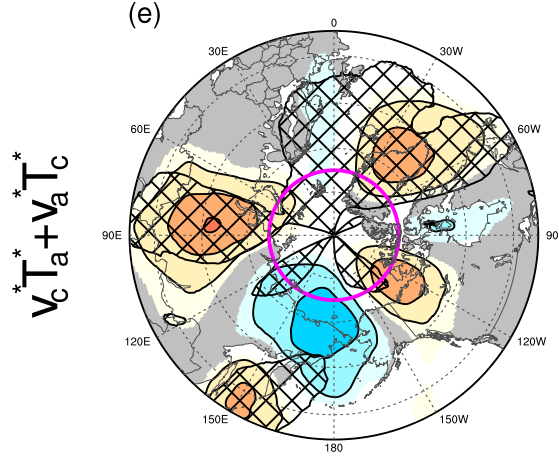

(g)

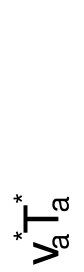

B25

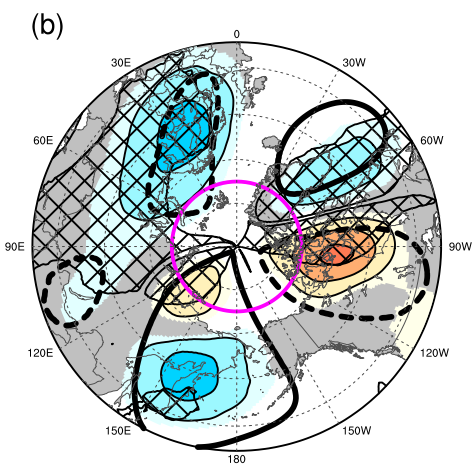

(d)

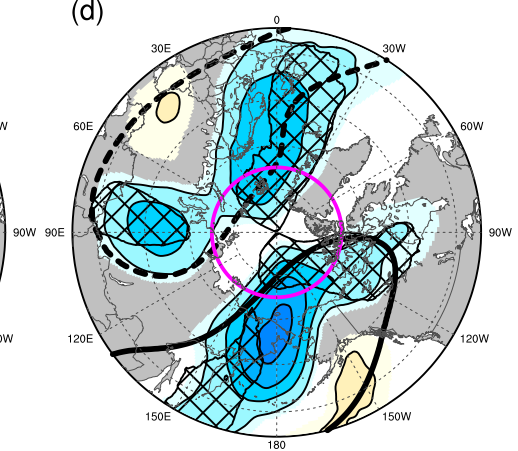

(f)

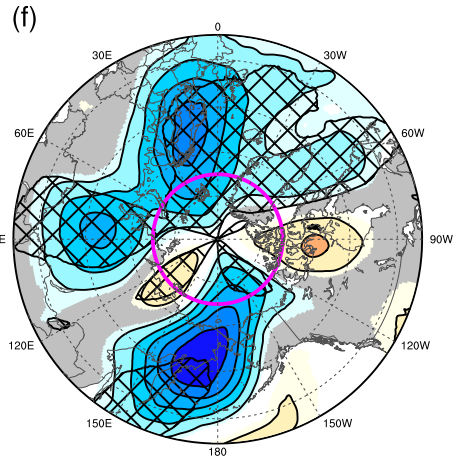

(h)

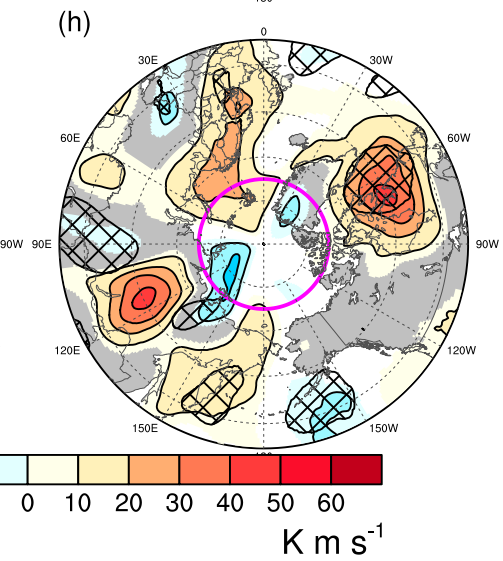

FIG. 12. As in Fig. 11, but for west Pacific blocks. 
T25

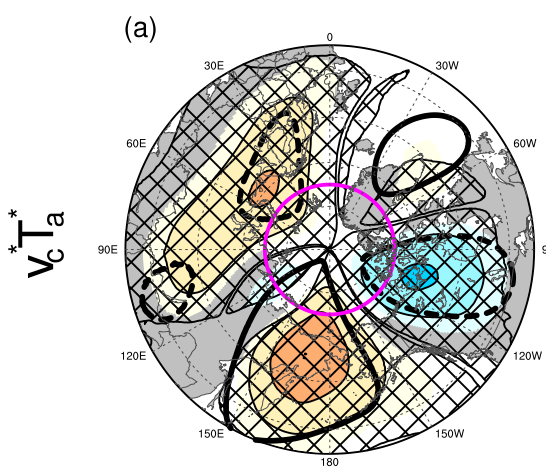

(c)

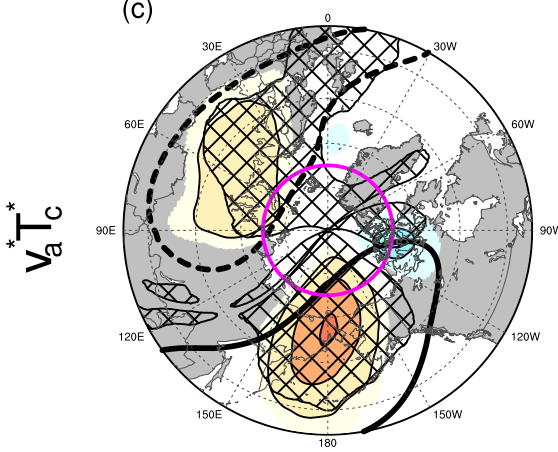

(e)

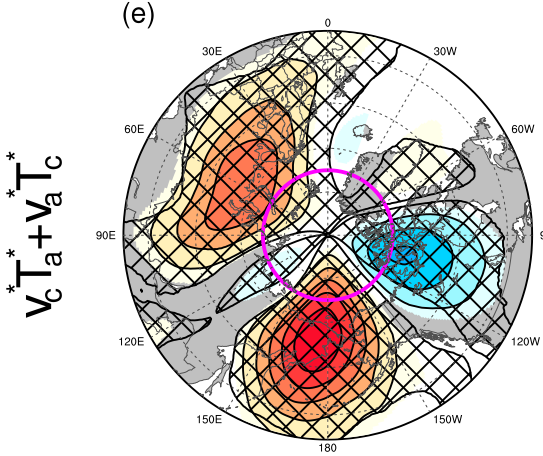

(g)

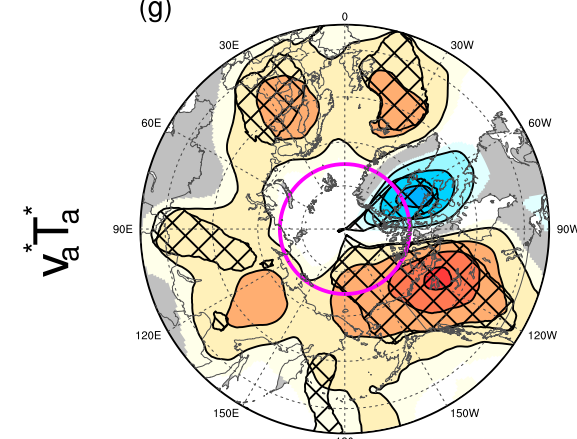

B25

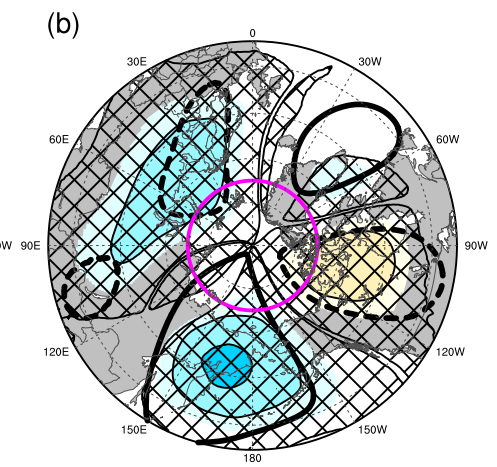

(d)

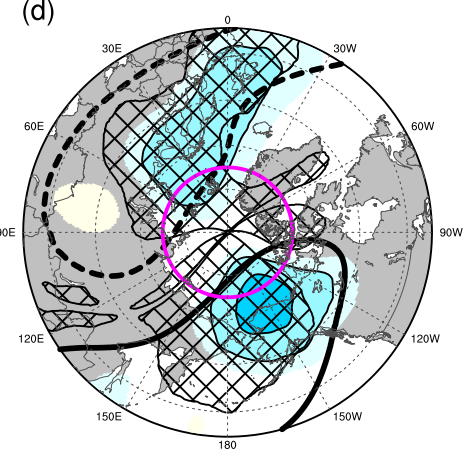

(1)

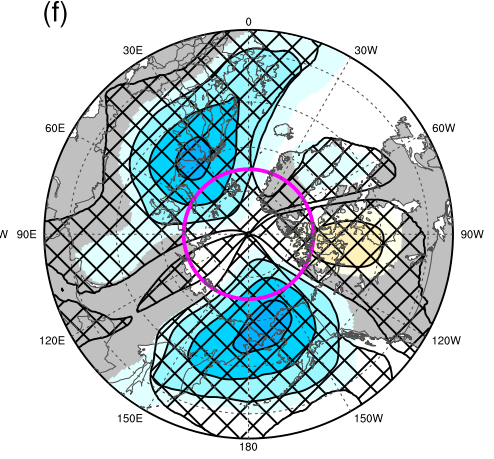

(i)

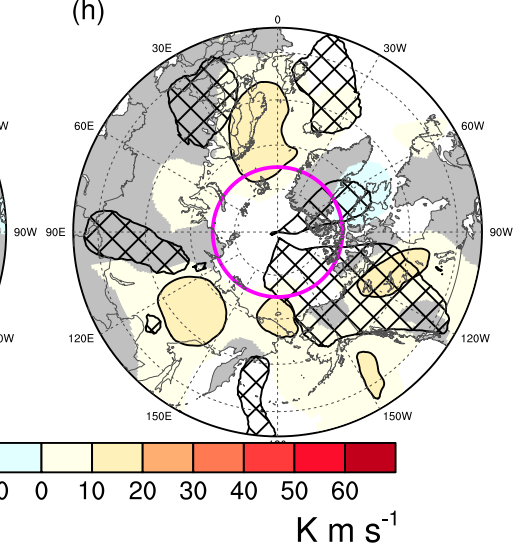

FIG. 13. As in Fig. 11, but for Atlantic bombs. 
T25

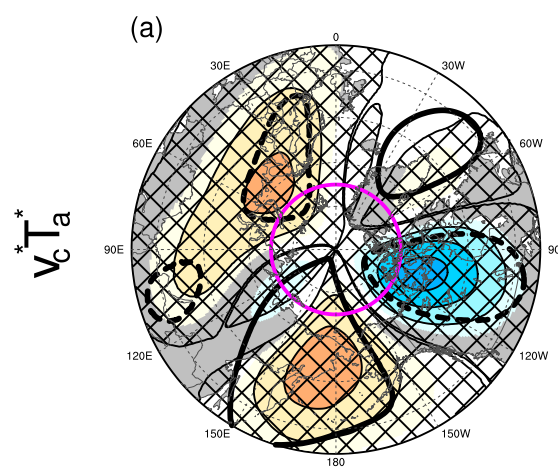

(c)

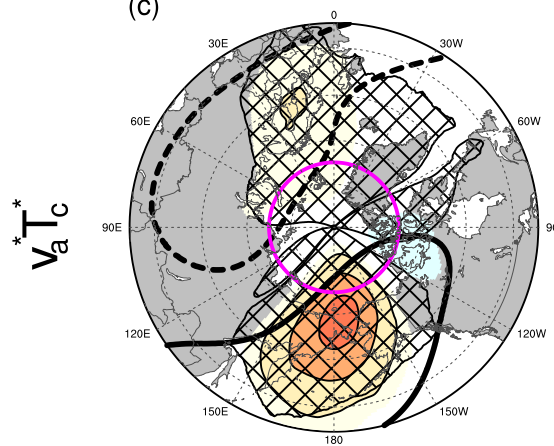

(e)

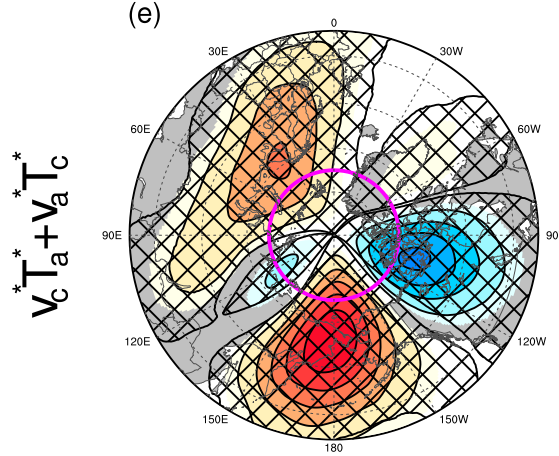

(g)

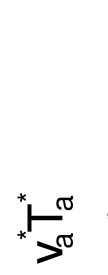

B25

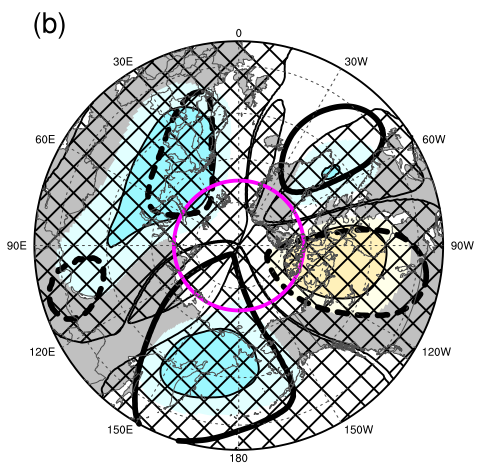

(d)

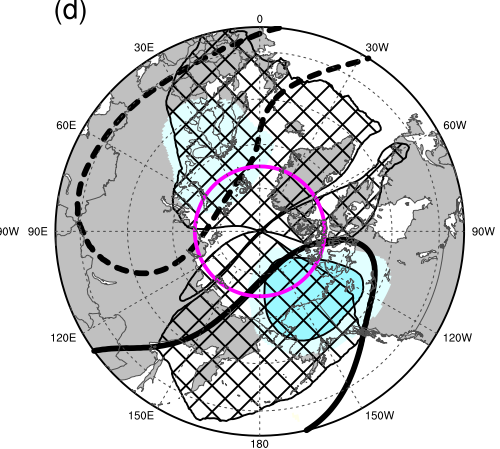

(f)

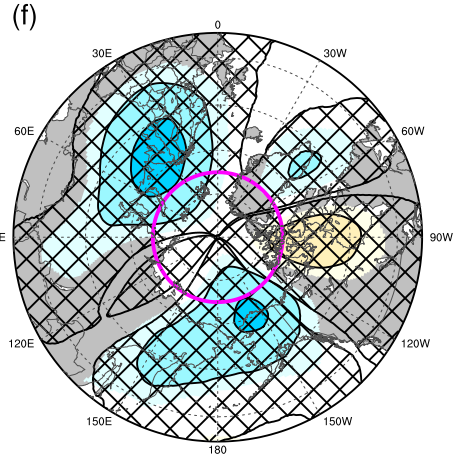

(h)

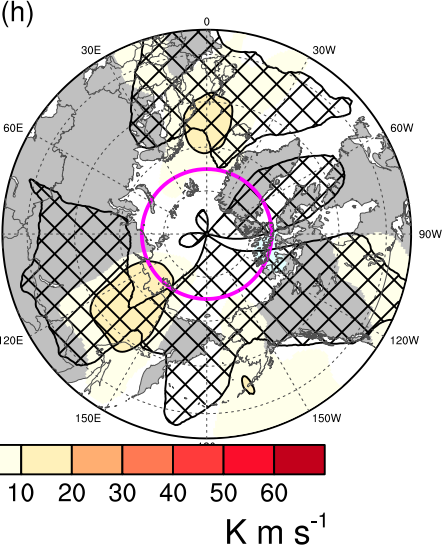

FIG. 14. As in Fig. 11, but for west Pacific bombs. 


\section{(a) T25 events}

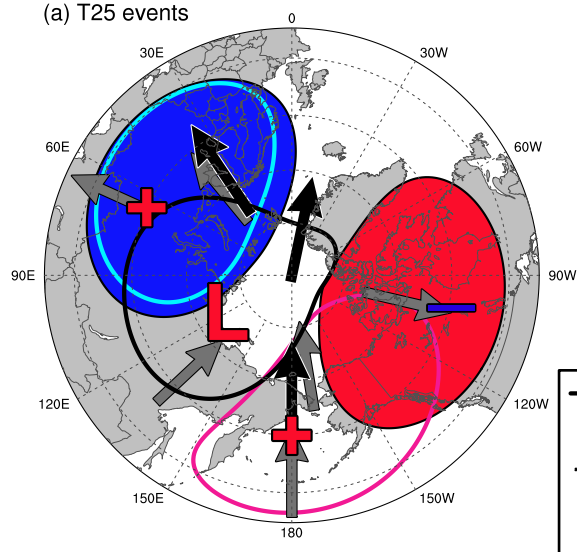

$100 \mathrm{hPa}$ at day +5

Example geopotential
height contour
Approximate location

of low

Meridional wind

Negative Negative temperature anomaly Positive temp
anomaly Negative climatologica

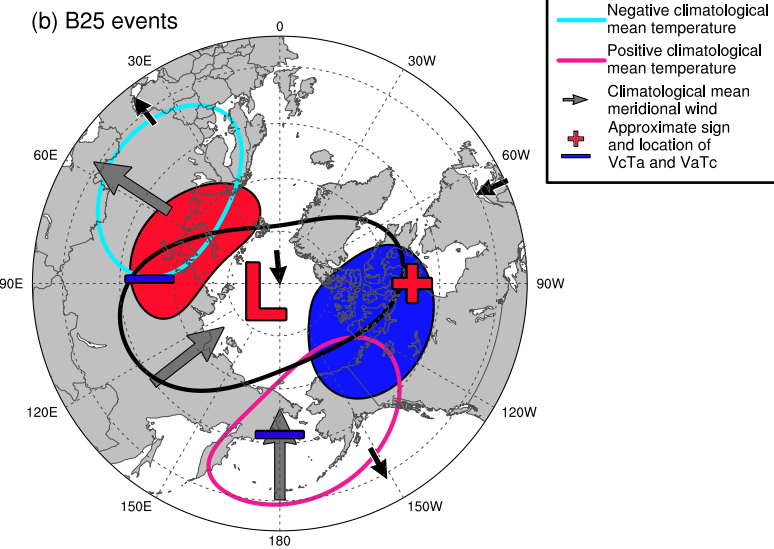

FIG. 15. Conceptual diagram of the 100 -hPa features at day +5 after onset of the synoptic events in the (a) T25 and (b) B25 groups. The symbols are as described in the legend.

meridional wind field is driving the negative heat flux anomaly signal.

\section{Blocks following bombs}

An important caveat to consider in this analysis is that blocks and bombs are not necessarily independent events, thus this section examines bombs and blocks that occur in sequence. For this analysis, the case-list described in Attard and Lang (2019) of bomb-block events is utilized. To summarize, bombblocks were defined as a block that occurred within $60^{\circ}$ longitude and 5 days of a point on the track of any identified bomb. There are 329 bombs and 183 blocks that make up the 183 identified bomb-blocks. Of these events, there is a blocking frequency maximum in Europe and a bomb-onset frequency maximum in the west Pacific (Table 1).

Of the 329 bombs that are followed by a block, 98 occurred in the Atlantic sector and 125 occurred in the west Pacific, which together accounts for $68 \%$ of all bombs followed by blocks (Table 1). The list of bombs used to create the list of bomb-blocks does not have the temporal/strength filter applied to the total list of bombs that was included in this study, thus some of the bombs on the bomb-block list are not considered in the T25 or B25 bomb events. However, of the $82 \mathrm{~T} 25$ Atlantic bombs, $21(25.7 \%)$ were identified on the bomb-block list and of the 82 B25 Atlantic bombs, only 10 (12.2\%) were identified on the bomb-block list (Table 4). Of the 169 T25 and 169 B25 west Pacific bombs, less than 15\% in either group were identified on the bomb-block list (Table 4).

Of the 19 T25 European blocks, 15 (79.9\%) were bomb-blocks while 13 (68.4\%) of the 19 B25 European blocks were bomb-blocks (Table 5). The opposite is true for west Pacific bomb-blocks. Of the 14 T25 west Pacific blocks, 7 (50.0\%) were bomb-blocks while 9 (64.3\%) B25 west Pacific blocks were bomb-blocks (Table 5). In general, the sign of the tropopause heat flux anomaly following bomb-blocks in the European and west Pacific regions are shifted in opposite directions, consistent with the mean of all European and west Pacific blocks.

\section{Conclusions}

This analysis considered the cool-season 100- and 250-hPa heat flux anomaly, defined as the zonal-mean meridional eddy heat flux anomaly with respect to the climatological mean. Specifically the analysis focused on extreme heat flux anomalies that followed the onset of blocking anticyclones (i.e., blocks) and extratropical cyclones that rapidly deepen (i.e., bombs), to achieve three goals:

- Goal 1: Quantify the distribution of all cool-season heat flux anomalies.

The results showed that the sign of the mean heat flux anomaly following synoptic events was dependent on both the month and region in which the event occurred. The range of heat flux anomalies following blocks and bombs is large and the maxima and minima for all events are comparable (Fig. 1). Blocks and bombs in each region were further partitioned according to the top and bottom quartile (i.e., T25 and B25) of the attendant 11-day-average $100-\mathrm{hPa}$ heat flux anomaly and 250-hPa standardized heat flux anomaly. The T25 and B25 events were analyzed in the context of extreme 100-hPa heat flux anomaly events from all cool-season days (i.e., the top and bottom 10\%). European blocks and west Pacific bombs, which, on average, are followed by positive heat flux anomaly, are often associated with the top heat flux events. West Pacific blocks and Atlantic bombs, which, on average, are followed 
TABLE 4. The number of bombs in bomb-blocks in the top (T25) and bottom (B25) quartiles of all Atlantic and west Pacific bombs based on the 100-hPa heat flux anomaly.

\begin{tabular}{ccc}
\hline \hline & Atlantic $(n=82)$ & West Pacific $(n=169)$ \\
\hline T25 & $21(25.7 \%)$ & $16(9.5 \%)$ \\
B25 & $10(12.2 \%)$ & $25(14.8 \%)$ \\
\hline
\end{tabular}

by negative heat flux anomaly, are often associated with bottom heat flux events. This relationship between the 100-hPa T25 and B25 events and the unique 100-hPa extreme heat flux events showed that most top and bottom unique heat flux events were associated with a synoptic event. This relationship is similar, but weaker, when considering the $250-\mathrm{hPa}$ standardized heat flux anomaly and the unique $100-\mathrm{hPa}$ standardized heat flux anomaly events.

- Goal 2: Determine the location of blocks and bombs that are associated, on average, with the most extreme heat flux anomalies.

The results are consistent with Nishii et al. (2011) and show that European blocks $(n=77)$ are associated with a statistically significant positive heat flux anomaly, while west Pacific blocks $(n=54)$ are followed by a statistically significant negative heat flux anomaly. Conversely, bombs that occurred in the Atlantic $(n=328)$ were associated with a statistically significant negative heat flux anomaly and bombs that occurred in the west Pacific $(n=676)$ were associated with a statistically significant positive heat flux anomaly. The results of this research support previous work that has shown the important relationship between blocking location and 100- or 200-hPa heat flux (e.g., Martius et al. 2009; Nishii et al. 2011; Colucci and Kelleher 2015). However, the results also highlight that within each region, individual blocks and bombs can be followed by either positive or negative heat flux anomaly, suggesting that the location of the synoptic event alone is not enough information to determine the sign of the attendant heat flux anomaly. The results also show that the evolution of the 250-hPa heat flux anomaly surrounding event onset is similar and of the same sign of the $100-\mathrm{hPa}$ heat flux anomaly.

- Goal 3: Compare the synoptic- and planetary-scale structures of blocks and bombs that (i) occur in the same region but are followed by opposite signed heat flux anomalies and (ii) have the same sign of heat flux anomalies.

For this part of the analysis, the heat flux anomaly was decomposed following Nishii et al. (2009). In this decomposition, the meridional wind and temperature
TABLE 5. As in Table 4, but for blocks in bomb-blocks in Europe and the west Pacific.

\begin{tabular}{ccc}
\hline & Europe $(n=19)$ & West Pacific $(n=14)$ \\
\hline T25 & $15(79.9 \%)$ & $7(50.0 \%)$ \\
B25 & $13(68.4 \%)$ & $9(64.3 \%)$ \\
\hline
\end{tabular}

fields were partitioned into their climatological and anomalous parts to quantify the interaction of the: 1) climatological and anomalous waves and 2) the anomalous waves with each other. Within each region, the temporal analysis showed that the interaction of the anomalous waves with the climatological waves was an important indicator of the sign of the $100-\mathrm{hPa}$ zonalmean heat flux anomaly. This is consistent with Woollings et al. (2010), who showed that the projection of European blocks onto the stationary wave pattern is important for increasing upward WAF. The distributions of the 100-hPa heat flux anomaly decomposition terms were spatially similar for all events in the same quartile of heat flux anomaly, across the event types and locations. All T25 events, except for west Pacific blocks, were associated with an 100-hPa polar vortex displaced toward the Eastern Hemisphere and corresponding positive centers of heat flux anomalies near the date line and $60^{\circ} \mathrm{E}$. These heat flux maxima were a result of the interaction between the climatological and anomalous waves. The B25 events, however, were associated with an 100-hPa polar vortex centered over the pole and negative heat flux anomalies near the date line and $90^{\circ} \mathrm{E}$ that were the result of the interaction between the climatological and anomalous waves. Finally, the T25 groups were associated with a baroclinic vertical wave structure within the troposphere and stratosphere while the B25 groups were associated with a barotropic vertical wave structure.

The analysis showed that blocks and bombs could both be followed by heat flux anomalies that were in the \pm 10 th percentile of the climatological heat flux anomaly. In an effort to examine whether consecutive blocks and bombs could be linked to extreme heat flux anomalies, bomb-blocks were identified. With respect to consecutive events, the results showed that most T25 European blocks are bomb-blocks, while just under two thirds of the west Pacific bomb-blocks had heat flux anomalies in the B25 group of west Pacific blocks. There was no clear relationship when considering the bombs in bomb-blocks.

The statistical significance of the stratospheric structure at the onset of blocks and bombs with respect to the sign of heat flux anomaly following these synoptic events is consistent with de la Cámara et al. (2017). de la Cámara et al. (2017) performed a modeling study to 
elucidate the role of the precursor stratospheric conditions in producing an SSW. They found that for displacement SSWs (i.e., WN1 SSWs), different initial conditions in the stratosphere 21 days before an SSW alters both the evolution of the 100-hPa vertical WAF and, ultimately, the SSW outcome. de la Cámara et al. (2017) argue that the increase in heat flux anomaly prior to an SSW may then not be from anomalous tropospheric forcing but a result of stratospheric processes. As there was a strong relationship between the T25 and B25 events identified by the $250-$ and $100-\mathrm{hPa}$ standardized heat flux anomaly, the results presented in this paper emphasize the importance of both the tropospheric and the stratospheric wave structures in the resultant sign of heat flux anomaly. Additionally, the results emphasize that both blocks and bombs can substantially impact the tropopause flow in such a way to be favorable for upward WAF.

- A comment on the relationship between T25 and B25 events and SSWs

Because a goal of this research is to understand the potential for synoptic events to be associated with the upward WAF that can precede SSWs, this section considers the number of synoptic events that occurred in conjunction with an SSW. This analysis utilized the Charlton and Polvani (2007) definition of SSWs, which identifies SSWs at $10 \mathrm{hPa}$ and $60^{\circ} \mathrm{N}$ if the daily averaged zonal-mean zonal wind, hereafter $\left[u_{60}\right]$, reversed to easterly. To ensure unique SSWs were identified and no final warmings were included, the following two criteria must also be met: the $\left[u_{60}\right]$ must 1 ) recover to westerly for at least 20 days between events and 2) return to westerly for at least 10 consecutive days. Utilizing this definition identifies 22 SSWs in the MERRA-2 dataset from 1980 to 2015. The onset date of the SSW is defined as the first date that $\left[u_{60}\right]$ is negative.

With respect to the $100-\mathrm{hPa}$ T25 and B25 events, only one B25 event occurred within the 10 days prior to an SSW (a west Pacific bomb). With respect to the T25 blocks, only four T25 European blocks (21\%) preceded an SSW and no T25 west Pacific blocks preceded an SSW. Though more T25 bombs preceded SSWs (9 Atlantic bombs and 19 west Pacific bombs), with respect to the total number of $\mathrm{T} 25$ bombs in each location this only accounted for $11 \%$ of the T 25 events. When conducting this same analysis with the lists of T25 and B25 250-hPa standardized anomalies, the numbers are quite similar. The same four European blocks were also in the T25 category based on the $250-\mathrm{hPa}$ standardized anomalies and there were no T25 west Pacific blocks. The percentage of Atlantic and west Pacific T25 bombs were slightly smaller than the $100-\mathrm{hPa} \mathrm{T} 25$ bombs, at $9 \%$ and $7 \%$, respectively. There were also a few B25 bombs prior to SSWs, but those made up only $2 \%$ of their respective categories. This suggests that there are more synoptic events associated with positive $250-$ or $100-\mathrm{hPa}$ heat flux anomalies than there are SSWs. This can be partially attributed to the strict definitions of both synoptic events and SSWs utilized in this study. However, the similarities in the number of T25 events followed by SSWs when defining T25 events by $250-$ or $100-\mathrm{hPa}$ heat flux anomaly suggests that the WAF identified at $100 \mathrm{hPa}$ following synoptic events that precede SSWs does originate near the tropopause.

Acknowledgments. This work was supported by NASA Headquarters under the NASA Earth and Space Science Fellowship Program Grant NNX16AO01H awarded to the first author and National Science Foundation Award 1547814 and NOAA Award NA16OAR4310068 granted to the second author. This research contributes to the efforts of the NOAA MAPP S2S Prediction Task Force. The MERRA-2 dataset is listed in the references and can be found at https://disc.sci.gsfc.nasa.gov/uui/ datasets/M2I3NPASM $\backslash$ V5.12.4/summary?keywords=। $\% 22$ MERRA-2 $\% 22$.

\section{REFERENCES}

Attard, H. E., and A. L. Lang, 2019: The impact of tropospheric and stratospheric tropical variability on the location, frequency, and duration of cool-season extratropical synoptic events. Mon. Wea. Rev., 147, 519-542, https://doi.org/10.1175/ MWR-D-18-0039.1.

Baldwin, M. P., and T. J. Dunkerton, 2001: Stratospheric harbingers of anomalous weather regimes. Science, 294, 581-584, https://doi.org/10.1126/science.1063315.

Birner, T., and J. R. Albers, 2017: Sudden stratospheric warmings and anomalous upward wave activity flux. SOLA, 13A , 8-12, https://doi.org/10.2151/sola.13A-002.

Charlton, A. J., and L. M. Polvani, 2007: A new look at stratospheric sudden warnings. Part I: Climatology and modeling benchmarks. J. Climate, 20, 449-469, https://doi.org/10.1175/JCLI3996.1.

Colucci, S. J., and M. E. Kelleher, 2015: Diagnostic comparison of tropospheric blocking events with and without sudden stratospheric warming. J. Atmos. Sci., 72, 2227-2240, https:// doi.org/10.1175/JAS-D-14-0160.1.

Coy, L., and S. Pawson, 2015: The major stratospheric sudden warming of January 2013: Analyses and forecasts in the GOES-5 data assimilation system. Mon. Wea. Rev., 143, 491510, https://doi.org/10.1175/MWR-D-14-00023.1.

_, S. Eckermann, and K. Hoppel, 2009: Planetary wave breaking and tropospheric forcing as seen in the stratospheric sudden warming of 2006. J. Atmos. Sci., 66, 495-507, https://doi.org/ 10.1175/2008JAS2784.1.

de la Cámara, A., J. R. Albers, T. Birner, R. R. Garcia, P. Hitchcock, D. E. Kinnison, and A. K. Smith, 2017: Sensitivity of sudden stratospheric warmings to previous stratospheric conditions. J. Atmos. Sci., 74, 2857-2877, https://doi.org/10.1175/JAS-D-170136.1. 
Díaz-Durán, A., E. Serrano, B. Ayarzagüena, M. Abalos, and A. de la Cámara, 2017: Intra-seasonal variability of extreme boreal stratospheric polar vortex events and their precursors. Climate Dyn., 49, 3473-3491, https://doi.org/10.1007/ s00382-017-3524-1.

Edmon, H. J., Jr., B. J. Hoskins, and M. E. McIntyre, 1980: Eliassen-Palm cross sections for the troposphere. J. Atmos. Sci., 37, 2600-2616, https://doi.org/10.1175/1520-0469(1980) $037<2600$ :EPCSFT $>2.0$. CO; 2 .

Gelaro, R., and Coauthors, 2017: The Modern-Era Retrospective Analysis for Research and Applications, version 2 (MERRA-2). J. Climate, 30, 5419-5454, https://doi.org/ 10.1175/JCLI-D-16-0758.1.

Global Modeling and Assimilation Office, 2015: MERRA-2 inst3_3d_asm_np: 3d,3-hourly,instantaneous,pressure-leve1,assimilation,assimilated meteorological fields v5.12.4. Goddard Earth Sciences Data and Information Services Center (GES DISC), Greenbelt, MD, accessed 1 August 2016, https:// doi.org/10.5067/QBZ6MG944HW0.

Hodges, K. I., 1994: A general method for tracking analysis and its application to meteorological data. Mon. Wea. Rev., 122, 2573-2586, https://doi.org/10.1175/1520-0493(1994)122<2573: AGMFTA $>2.0 . \mathrm{CO} ; 2$.

1995: Feature tracking on the unit sphere. Mon. Wea. Rev., 123, 3458-3465, https://doi.org/10.1175/1520-0493(1995)123<3458: FTOTUS $>2.0 . \mathrm{CO} ; 2$.

Holton, J. R., 2004: An Introduction to Dynamic Meteorology. 4th ed. Elsevier Academic Press, 535 pp.

Martius, O., L. M. Polvani, and H. C. Davies, 2009: Blocking precursors to stratospheric sudden warming events. $G e$ ophys. Res. Lett., 36, L14806, https://doi.org/10.1029/ 2009GL038776.
Nishii, K., H. Nakamura, and T. Miyasaka, 2009: Modulations in the planetary wave field induced by upward-propagating Rossby wave packets prior to stratospheric sudden warming events: A case study. Quart. J. Roy. Meteor. Soc., 135, 39-52, https://doi.org/10.1002/qj.359.

- - — , and Y. J. Orsolini, 2011: Geographical dependence observed in blocking high influence on the stratopsheric variability through enhancement and suppression of upward planetary-wave propagation. J. Climate, 24, 6408-6423, https:// doi.org/10.1175/JCLI-D-10-05021.1.

Polvani, L. M., and D. W. Waugh, 2004: Upward wave activity flux as a precursor to extreme stratospheric events and subsequent anomalous surface weather regimes. J. Climate, $\mathbf{1 7}$, 3548-3554, https://doi.org/10.1175/1520-0442(2004)017<3548: UWAFAA $>2.0 . \mathrm{CO} ; 2$.

Quiroz, R. S., 1986: The association of stratospheric warmings with tropospheric blocking. J. Geophys. Res., 91, 5277-5285, https:// doi.org/10.1029/JD091iD04p05277.

Rienecker, M. M., and Coauthors, 2011: MERRA: NASA's Modern-Era Retrospective Analysis for Research and Applications. J. Climate, 24, 3624-3648, https://doi.org/10.1175/ JCLI-D-11-00015.1.

Sanders, F., and J. R. Gyakum, 1980: Synoptic-dynamic climatology of the "bomb." Mon. Wea. Rev., 108, 1589-1606, https://doi.org/ 10.1175/1520-0493(1980)108<1589:SDCOT > 2.0.CO;2.

Tibaldi, S., and F. Molteni, 1990: On the operational predictability of blocking. Tellus, 42A, 343-365, https://doi.org/10.3402/ tellusa.v42i3.11882.

Woollings, T., A. Charlton-Perez, S. Ineson, A. G. Marshall, and G. Masato, 2010: Associations between stratospheric variability and tropospheric blocking. J. Geophys. Res., 115, D06108, https://doi.org/10.1029/2009JD012742. 